\title{
Final Analytical Results from the Examination of Corrosion on Sections of Corrosion Probe Removed from Tank 241-AN-107 on August 10, 2006
}

\author{
G. A. Cooke and J. B. Duncan \\ ClI2M Hill Hanford Group, Inc. \\ Richland, WA 99352 \\ U.S. Department of Energy Contract DE-AC27-99RL14047 \\ $\begin{array}{lll}\text { EDT/ECN: } & \text { DRF } & \text { UC: } \\ \text { Cost Center: } & 7 S 110 & \text { Charge Code: } \\ \text { B\&R Code: } & & \text { Total Pages: } 54\end{array}$
}

Key Words: Tank 241-AN-107, crevice, slress, pitting, corrosion, optical microscopy, scanning electron microscopy, C-ring, bullet, electrochemical, noise, probe, specimens, analytical results, cxamination, macrophotography, photomicrograph, detector 1, detector 2, detector 3, detector 4, nonstressed, stressed, pre-stressed, disassembly, hot cell

Abstract: Tank farms Operations removed an electrochemical noise probe from Tank 241-AN-107. In the field, the probe was cut into four sections, wrapped, and placed in a 55-gallon drum. This drum was delivered to the 222-S Laboratory. The 222 S Laboratory unpackaged the sections of the AN-107 electrochemical noise probe and examined the material for evidence of corrosion. Each of the four sections containcd three $\mathrm{C}$-ring and three bullet specimens. The specimens were examined for pitting corrosion, crevice corrosion, and stress corrosion cracking. No evidence of stress corrosion cracking was found in the stressed C-ring specimens. Minor pitting was evident on some surfaces. Crevice corrosion was the dominant type of corrosion observed.

TRADEMARK DISCLAIMER. Reference herein to any specific commencial product, process, or service by trade name, trademark, manufacturer, or otherwlse, does not necessarly constitute or imply its endorsement, recommendation, or favoring by the United States Government or any agency thereof or its contractors or subcontractors.

Printed in the United States of America. To obtaln coples of this document, contact: Document Control Services, P.O. Box 950, Mailstop H6-08, Richland WA 99352, Phone (509) 372-2420; Fax (509\} 376-4989.
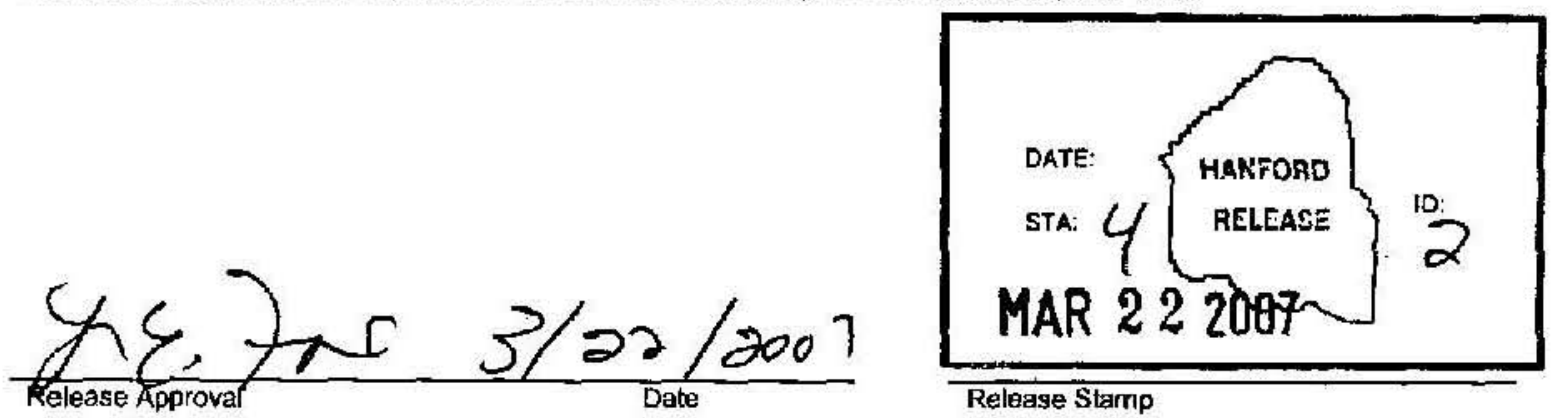

Approved For Public Release 
RPP-RPT-32425

Revision 0

\title{
FINAL ANALYTICAL RESULTS FROM THE EXAMINATION OF CORROSION ON SECTIONS OF CORROSION PROBE REMOVED FROM TANK 241-AN-107 ON AUGUST 10, 2006
}

\author{
G. A. Cooke \\ J. B. Duncan \\ CH2M HILL Hanford Group, Inc.
}

Date Published

March 2007

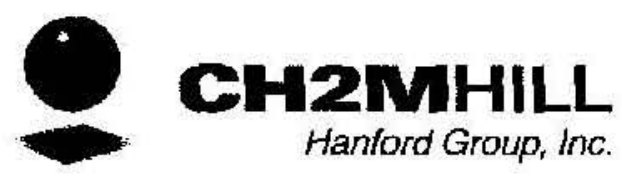

Prepared for the U.S. Department of Energy Office of River Protection 


\section{CONTENTS}

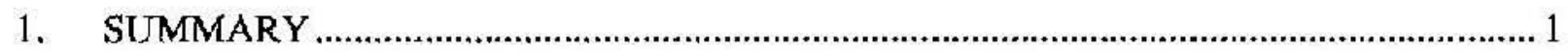

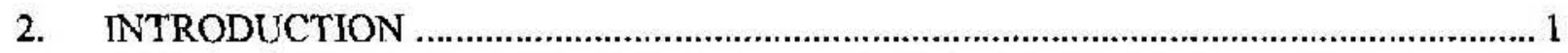

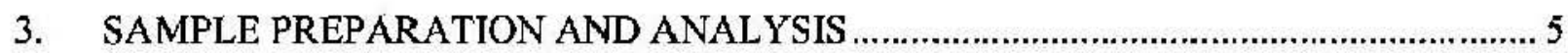

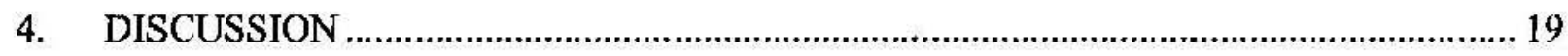

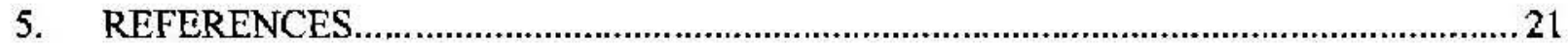

APPENDIX A. Image Names and Locations from the Examination of 241-AN-107 Electrochemical Noise Probe Specimens.........................................................A-1

\section{List of Figures}

Figure 1. General View of Detector 1 During Disassembly in I lood......................................... 3

Figure 2. Removal of Cross Bar from Detector 4 in Hot Cell. ................................................. 3

Figure 3. General View of Detector 2 During Disassembly in Hot Cell..................................... 4

Figure 4. General View of Detector 3 During Disassembly in Hot Cell.................................... 4

Figure 5. General View of Detector 4 During Disassembly in Hot Cell.................................... 5

Figure 6. Microphotograph (10 times) of Specimen S06R001024, Detector 1 Bullet, Uncleaned, Scale Bar $=1 \mathrm{~mm}$

Figure 7. Macrophotograph Specimen S06R001027, Detector 1, Stressed C-Ring, Uncleaned... 7 Figure 8. Macrophotograph Specimen S06R001033, Detector 2, Stressed C-Ring, Uncleaned... 7

Figure 9. Macrophotograph Specimen S06R001036, Detector 3, Bullet, Uncleaned, .................. 8

Figure 10. Macrophotograph Specimen S06R001038, Detector 3, Nonstressed C-Ring, Uncleaned,

Figure 11. Macrophotograph Specimen S06R001039, Detector 3, Stressed C-Ring, Uncleaned. 9

Figure 12. Macrophotograph Specimen S06R001042, Detector 4, Uncleaned........................... 9

Figure 13. Macrophotograph Specimen S06R001045, Detector 4, Stressed C-Ring, Uncleaned.

Figure 14. Macrophotograph of the Top of Vapor Space Nonstressed C-Ring S06R001028.... 12 Figure 15. SEM Microphotograph of the Top of Vapor Space Nonstressed C-Ring S06R001028.

Figure 16. SEM Photomicrograph of the Face of Pre-stressed Crack, Specimen S06R001027. 13 Figure 17. SEM Photomicrograph of the Face of Pre-stressed Crack, Specimen S06R001027. 14 Figurc 18. SEM Photomicrograph of the Face of Pre-stressed Crack, Specimen S06R001033. 14 Figure 19. SEM Photomicrograph of the Face of Pre-stressed Crack, Specimen S06R001045. 15 
Figure 20. SEM Photomicrograph of the Polished and Etched Surface of Pre-stressed Crack,

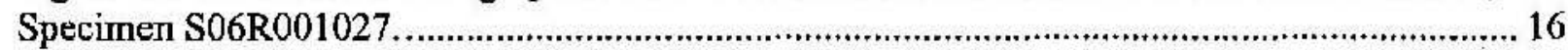

Figure 21. SEM Photomicrograph of the Polished and Etched Surface of Pre-stressed Crack,

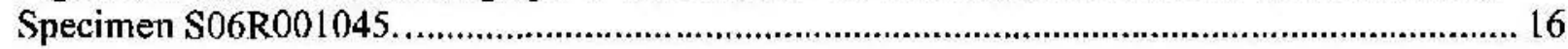

Figure 22. SEM Image of Pitted Arca on Outer Surface of Sample S06R001024 _.................. 17

Figure 23. SEM Image of Pilted Area Near Crevice Between C-Ring and Bolt Head on

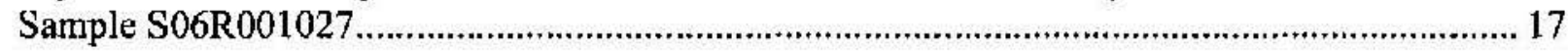

Figure 24. SEM Image of Pitted Area from End of Bolt on Sample SO6RO01028 .................. 18

Figure 25. SEM Imagc of Pitted Arca on Base of Sample S06R001042, .............................. 18

Figure 26. SEM Image of Pitted Area on Sample S06R001045 .......................................... 19

Figure 27. Bullet Sample S06R001023 ....................................................................... 20

\section{List of Tables}

Table 1. Tank 241-AN-107 Eloctrochemical Noise Coupon Identification. ................................ 2

Table 2. Weight Loss during Ammoniurn Citrate Cleaning.................................................. 11

Error! No table of figures entries found. 
RPP-RPT-32425, Rev. 0

\section{SUMMARY}

Tank Farms Operations removed an electrochemical noise probe from Tank $241-\Lambda \mathrm{N}-107$ (AN-107) on August 10,2006. In the field, the probe was cut into four sections, wrapped, and placed in a 55-gallon drum. This drum was delivered to the 222-S Laboratory on August 17,2006 . The 222-S Laboratory unpackaged the sections of the AN-107 electrochemical noise probe and examined the material for evidence of corrosion.

Each of the four sections contained three C-ring and three bullet specimens. Eight of these 24 specimens were removed and cleaned and examined to determine the extent of surface corrosion. The specimens were examined for pitting corrosion, crevice corrosion, and stress corrosion cracking. Macrophotography, optical microscopy (OM), and scanning electron microscopy (SEM) were used to document the disassembly process and to determine the extent of corrosion on each of these eight specimens.

No evidence of stress corrosion cracking was found in the stressed C-ring specimens. Minor pitting was evident on some surfaces. Crevice corrosion was the dominant type of corrosion observed.

Crevice corrosion, as a result of crevices created by poor probe design, was found at the top of the C-rings (where the threaded bolt met the $\mathrm{C}$-ring), at the base of the $\mathrm{C}$-ring (where the bolt contacted the $\mathrm{C}$-ring), and beneath the $\mathrm{C}$-ring and bullet specimens, where vapors and liquids penetrated behind the $\mathrm{O}$-ring seals and caused crevice corrosion between the detector elements and the O-rings. Pitting corrosion was found in isolated patches on the surfaces of some of the $\mathrm{C}$-rings and bullets. Both crevice and pitting corrosion were more noticeable on the specimcns that had been suspended in the vapor space of the tank (section 1), but were also present on somc of the other specimens recovered from the sections submerged in the waste.

Cleaned, cut, and polished sections revealed that pitting was not very well developed in the corroded areas. Pit depths from cross-section specimens were always less than $100 \mu \mathrm{m}$. Polished and/or freeze-fractured surfaces of the stressed C-rings showed no evidence for strcss corrosion cracking.

\section{INTRODUCTION}

This report describes the final results of the disassembly and examination of clectrochemical noise probe specimens removed from AN-107 on August 10, 2006. Preparation and analysis of these detectors were conducted at the 222-S Laboratory using internal letter 7S1 10-GAC-06-079 Reissue, "Transmit the Test Procedure for the Examination of Electrochemical Noise Probe Specimens to be Removed from Tank 241-AN-107, August 2006" (7S110-GAC-06-079 Reissue). The original laboratory test procedure was revised to address radiation dose issues that wcre discovered during the breakdown of the sample drum. 
The specimens were delivered to the 222-S Laboratory on August 17, 2006, as four detector sections containing six specimens each. The locations of the detectors and the three C-ring and three bullet specimens on each detector are identified in Table 1.

Table 1. Tank 241-AN-107 Electrochemical Noise Coupon Identification.

\begin{tabular}{|c|c|c|c|c|}
\hline Specimen Type & Location & Probe Section & Lab ID & Field ID \\
\hline Bullet & \multirow{6}{*}{ Vapor space } & \multirow[t]{6}{*}{ Detector 1} & SO6R001023 & a \\
\hline Bullet & & & SO6R001024 & $Z^{*}$ \\
\hline Bullet & & & S06R001025 & $Y$ \\
\hline C-ring & & & S06R001026 & $x$ \\
\hline Stressed C-ring & & & S06R001027 & $\mathrm{W}^{\mathrm{a}}$ \\
\hline C.-ring & & & S06R001028 & $v^{a}$ \\
\hline Bullet & \multirow[t]{6}{*}{ Supernatant } & \multirow[t]{6}{*}{ Detector 2} & S06R001029 & $\mathrm{U}$ \\
\hline Bullet & & & S06R001030 & $\mathrm{T}$ \\
\hline Bullet & & & S06R00103I & $\underline{\mathrm{S}}$ \\
\hline C-ring & & & S06R00t032 & $\overline{\mathbf{R}}$ \\
\hline Stressed C-ring & & & S06R001033 & $\overline{P^{3}}$ \\
\hline C-ring & & & S06R001034 & $N$ \\
\hline Bullet & \multirow[t]{6}{*}{ Supernatant } & \multirow[t]{6}{*}{ Detector 3} & S06R001035 & $\overrightarrow{\mathrm{M}}$ \\
\hline Bullet & & & S06R001036 & $\overline{\mathrm{L}^{2}}$ \\
\hline Bullet & & & SO6R001037 & $\mathrm{K}$ \\
\hline C-ring & & & S06R001038 & $\mathrm{J}$ \\
\hline Stressed C-ring & & & So6R001039 & $\mathrm{H}^{3}$ \\
\hline C-ring & & & SO6R001040 & $\mathrm{G}$ \\
\hline Bullet & \multirow[t]{6}{*}{ Saltcake } & \multirow[t]{6}{*}{ Detector 4} & S06R00104I & $\mathrm{F}$ \\
\hline Bullet & & & $\overline{S 06 R 00}+\overrightarrow{042}$ & $E^{a}$ \\
\hline Bullet & & & S06R001043 & $\bar{D}$ \\
\hline C-ring & & & S06R001044 & $\mathrm{C}$ \\
\hline Stressed C-ring & & & S06R001045 & $B^{a}$ \\
\hline C-ring & & & 50612001046 & $\bar{A}$ \\
\hline
\end{tabular}

${ }^{a}$ Specimens recovered for cleaning and analysis.

The specimens from Detector 1 (Figure 1), from the vapor space, were recovered in a fume hood using padded extension tools to avoid damaging the specimens. Detector 1 was removed from the drum, unwrapped in a hood, and all eight specimens from the detector arm were rinsed with inhibited water and placed in a dessicator.

Disassembly of the remaining sections in the hood was not possible due to high dose rates associated with the sections that had been submerged in the tank waste. These sections were loaded into a hot cell using step-by-step instructions (7S1 10-GAC-06-079 Reissue) that allowed the oversized sections to be passed through the airlock with both airlock doors open and a fiberreinforced bag taped to the outer wall of the hot cell to provide contamination control. In the hot cell, a rotary cutting tool was used to remove the metal bar covering the specimens to allow for removal with the hot cell manipulators (Figure 2). The detectors were rinsed with tap water 

during the hot cell breakdown.

Figure 1. General View of Detector 1 During Disassembly in Hood.

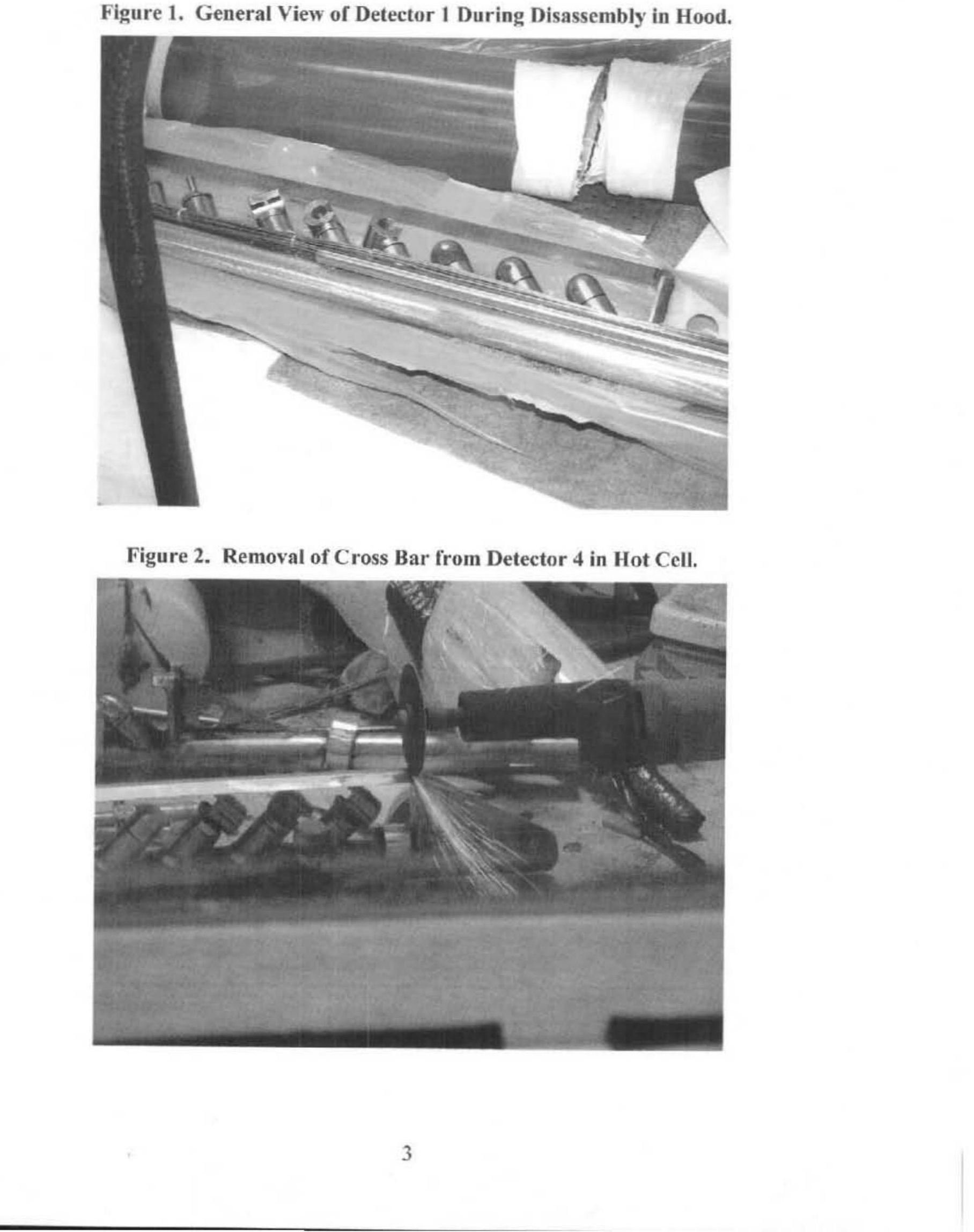

Figure 2. Removal of Cross Bar from Detector 4 in Hot Cell.

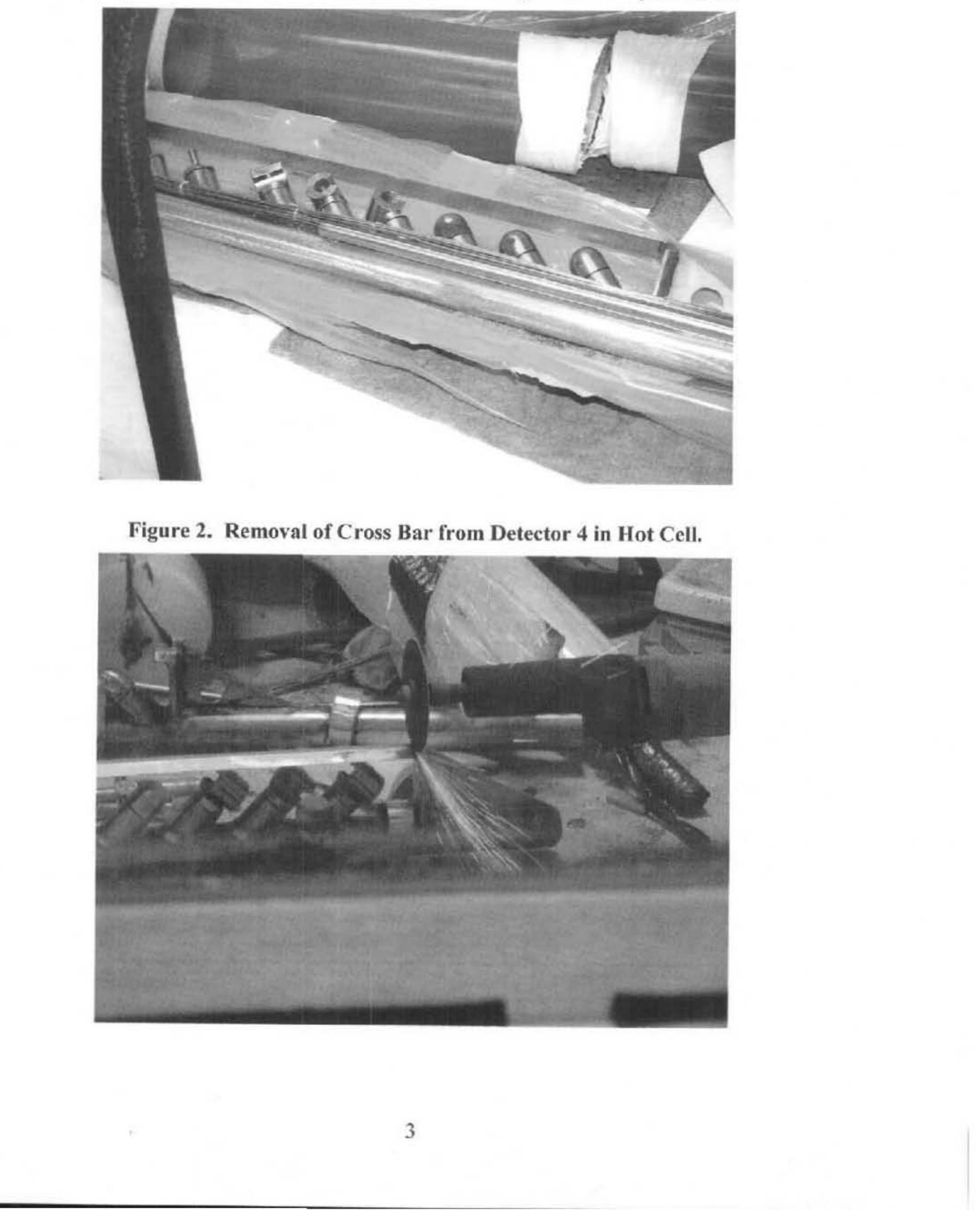
during the hot cell breakdown.

$$
\text { RPP-RPT-32425, Rev. } 0
$$
followed by inhibited water. Figure 3 through Figure 5 show the other three detector assemblies
RPP-RPT-32425, Rev. 0

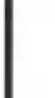
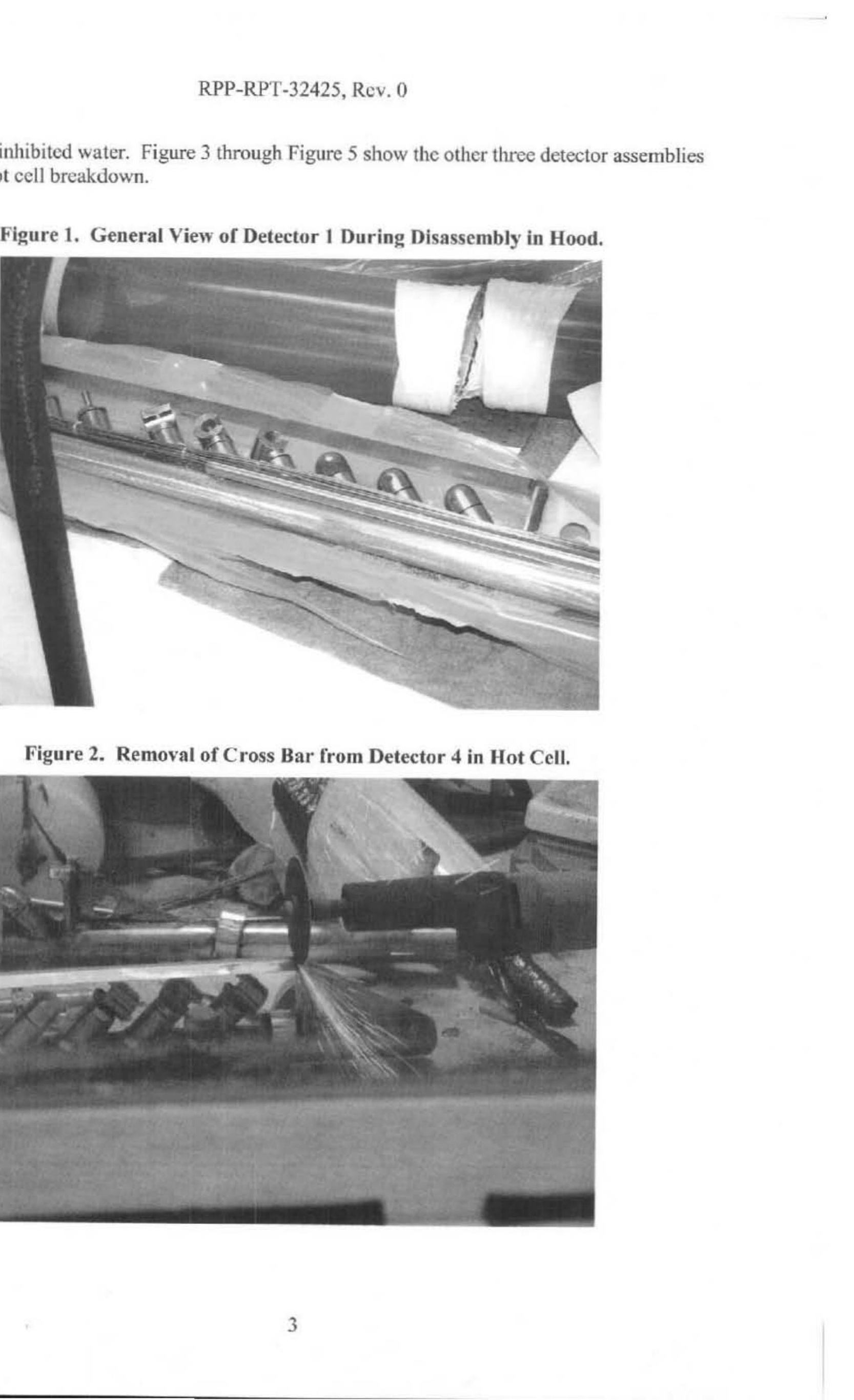


$$
\text { RPP-RPT-32425, Rev. } 0
$$

Figure 3. General View of Detector 2 During Disassembly in Hot Cell.

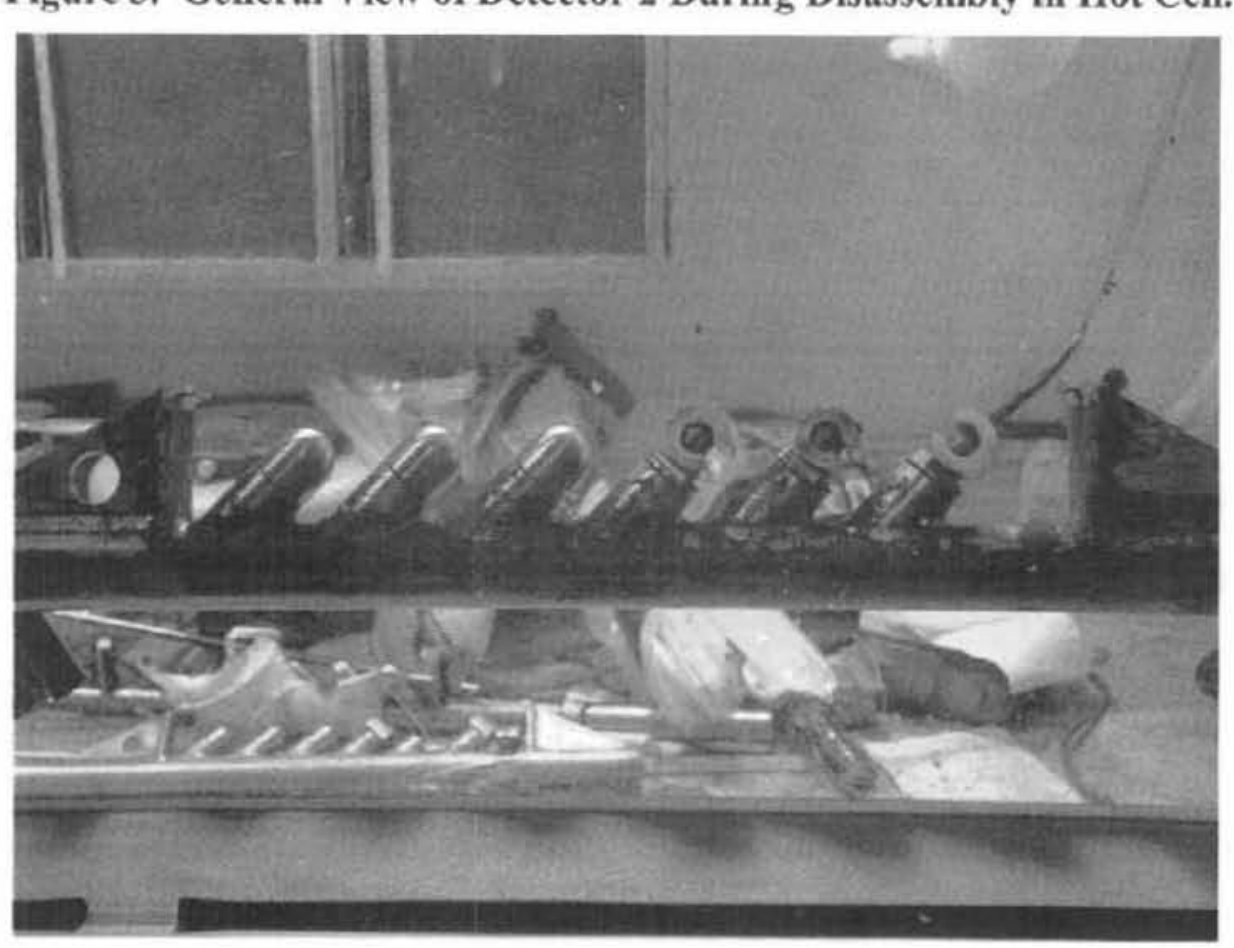

Figure 4. General View of Detector 3 During Disassembly in Hot Cell.

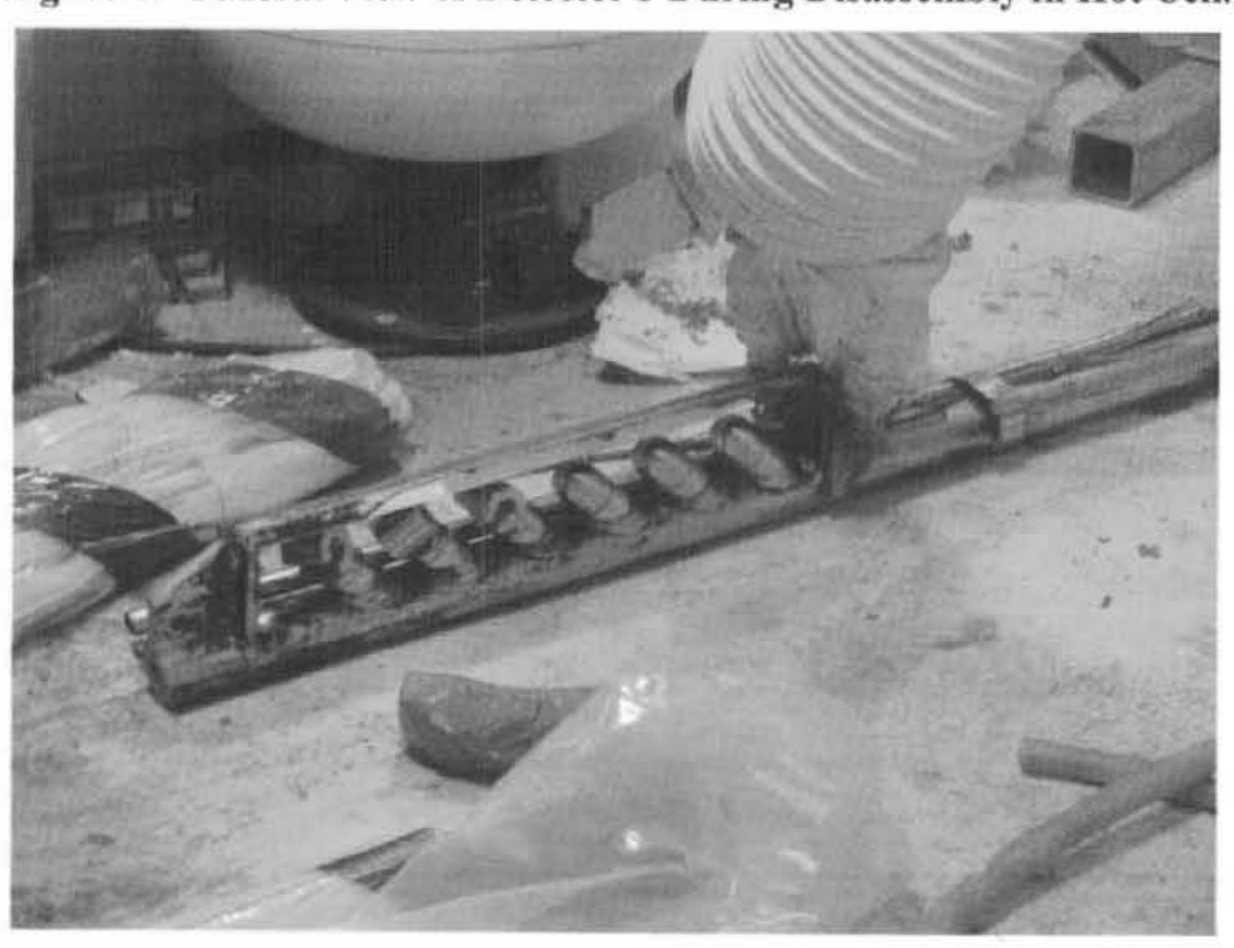


Figure 5. General View of Detector 4 During Disassembly in Hot Cell.

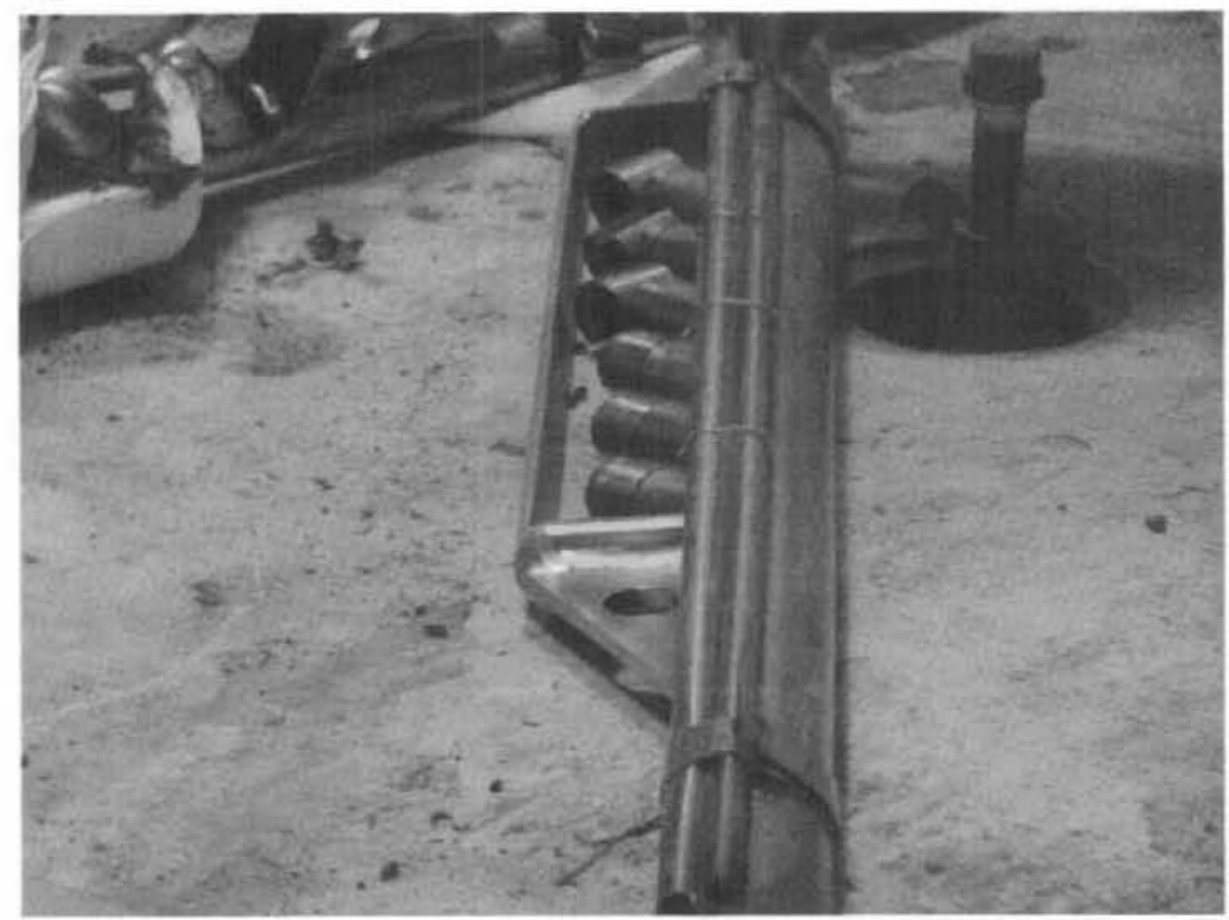

Selected C-ring and bullet specimens were retrieved from the detectors in the hot cell and then they were loaded out of the hot cell for analysis. Photographs taken during sample breakdown are listed in Appendix A. Many of these photos were processed through image analysis software to achieve color balance and to sharpen the images.

\section{SAMPLE PREPARATION AND ANALYSIS}

The selected specimens (identified in Table 1) were photographed, cleaned, and prepared for examination by reflected-light OM or SEM at the 222-S Laboratory. Selected features of the specimens were documented before and after cleaning. Representative macro and microphotographs are presented in Figure 6 through Figure 13. All photographs are tabulated in Appendix A. The digital images are posted on the chardocs share drive and are available on compact disk (CD).

The standard size $40-\mu \mathrm{m}$ grid measured approximately $10 \%$ too large under the operating conditions of the SEM (see Appendix A); therefore, all measurements recorded on SEM images and any measurements made using the image scale bars are approximately $10 \%$ too large. 


$$
\text { RPP-RPT-32425, Rev. } 0
$$

Figure 6. Microphotograph (10 times) of Specimen S06R001024, Detector 1 Bullet, Uncleaned, Scale Bar $=1 \mathrm{~mm}$.
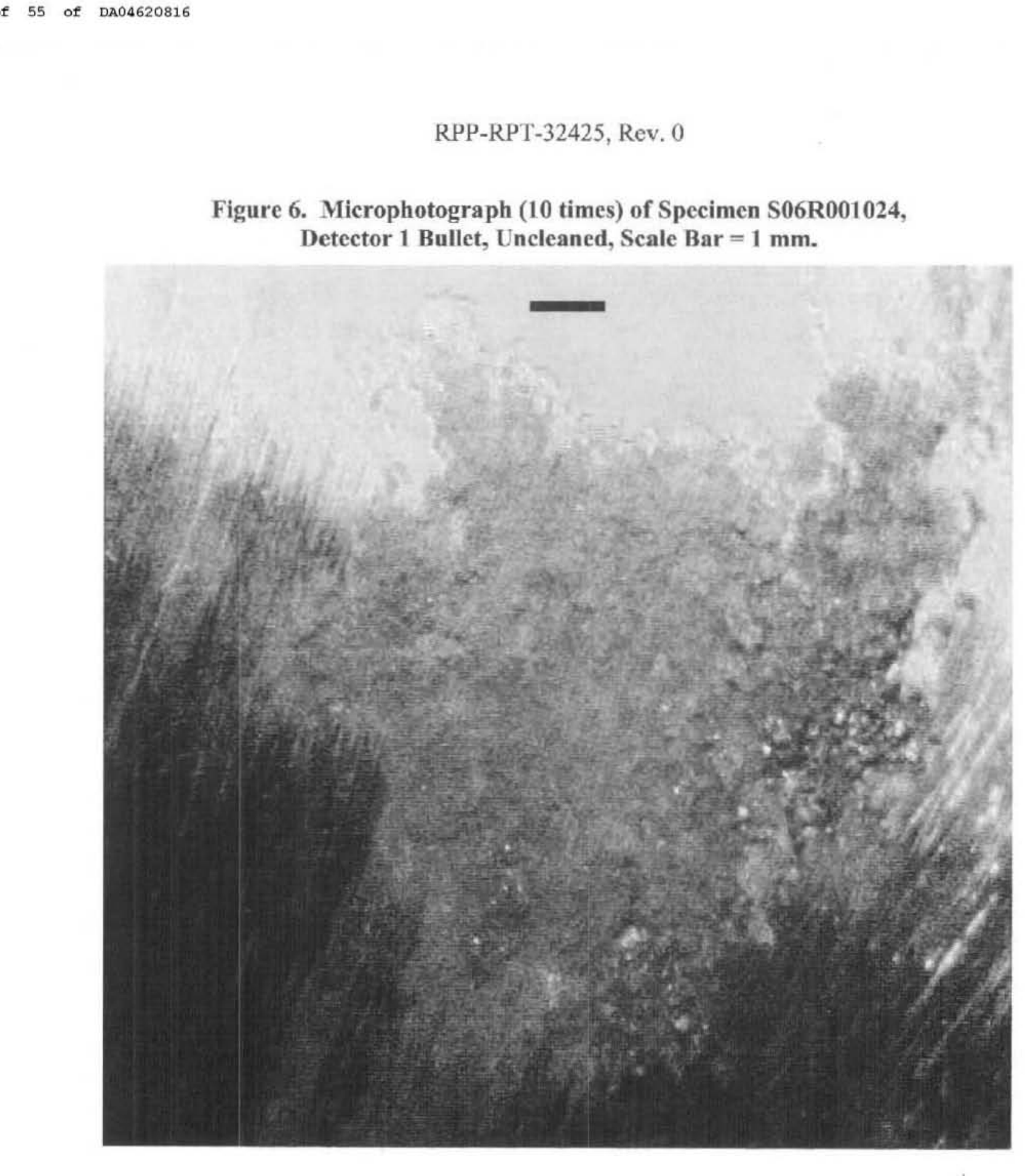

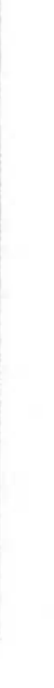

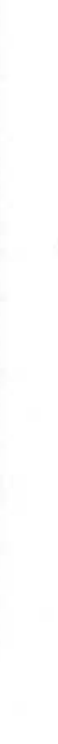

$-$ 


$$
\text { RPP-RPT-32425, Rev. } 0
$$

Figure 7. Macrophotograph Specimen S06R001027, Detector 1, Stressed C-Ring, Uncleaned.

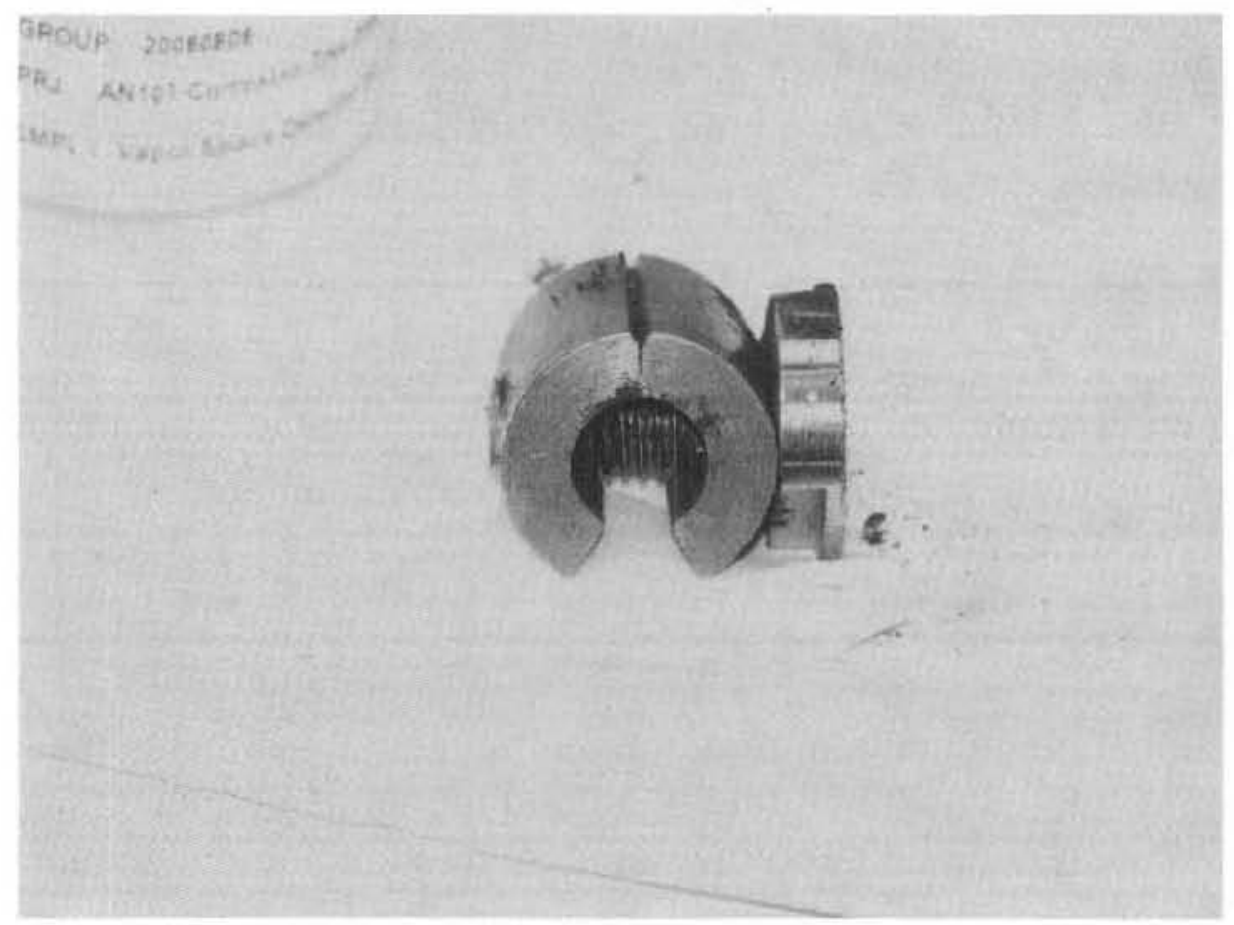

Figure 8. Macrophotograph Specimen S06R001033, Detector 2, Stressed C-Ring, Uncleaned.

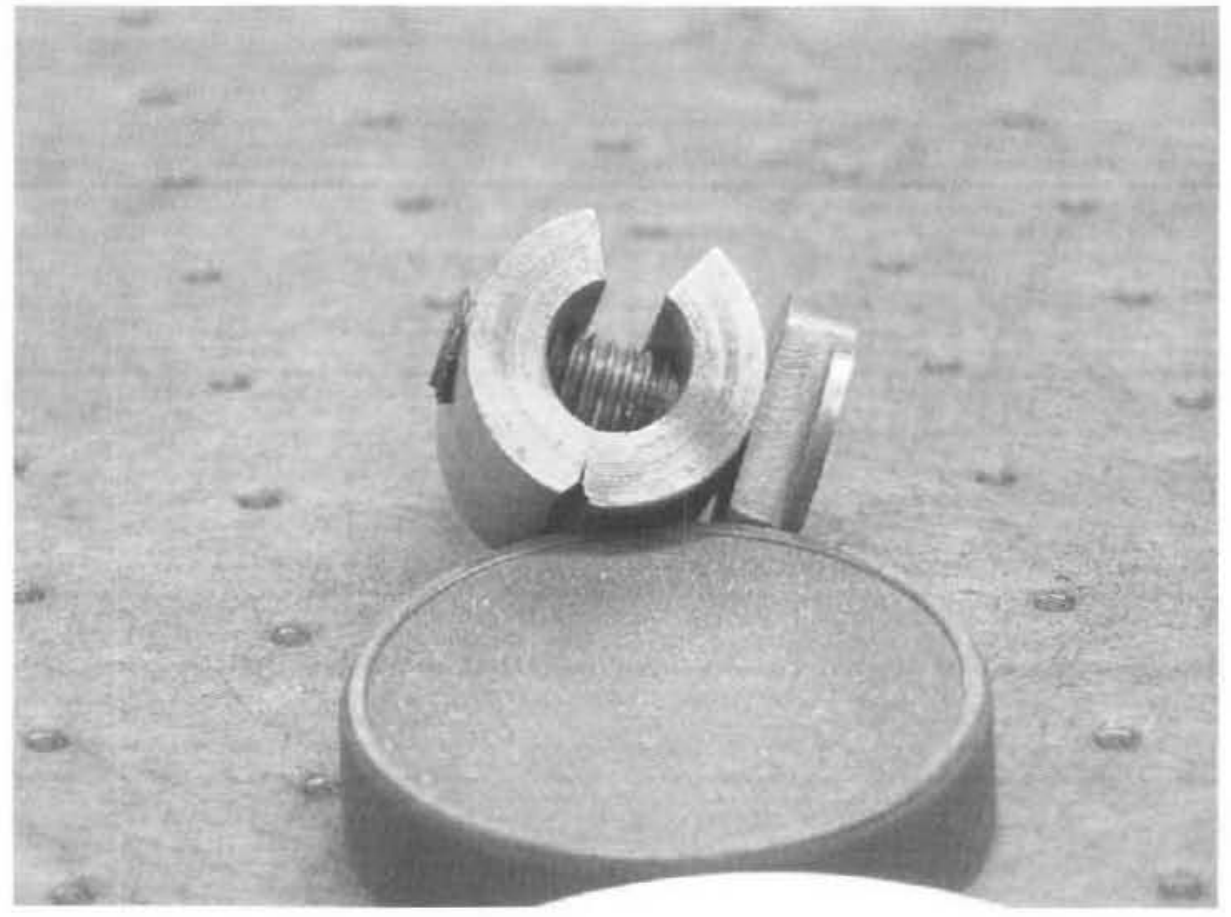


Figure 9. Macrophotograph Specimen S06R001036, Detector 3, Bullet, Uncleaned.

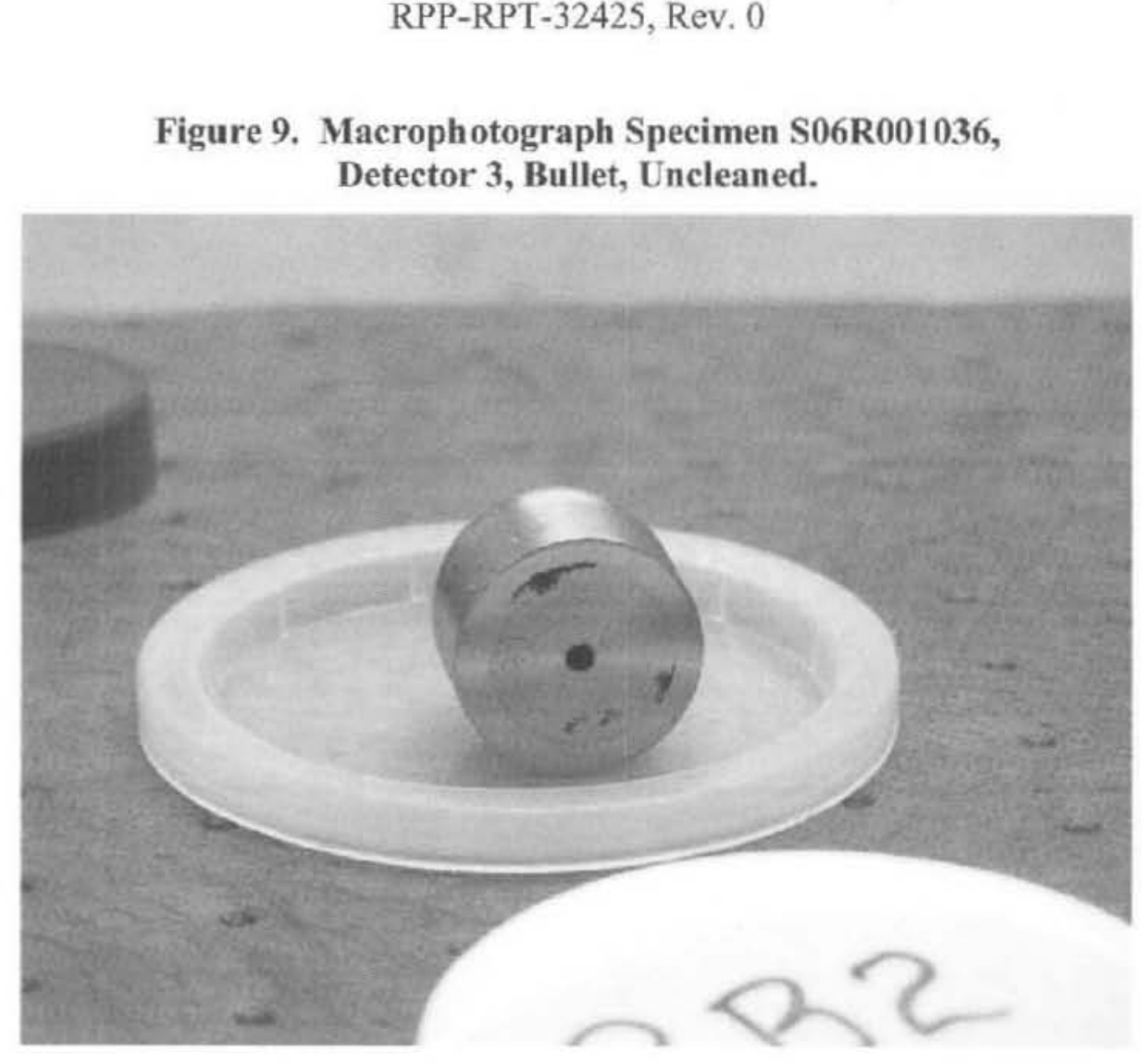

Figure 10. Macrophotograph Specimen S06R001038, Detector 3, Nonstressed C-Ring, Uncleaned.

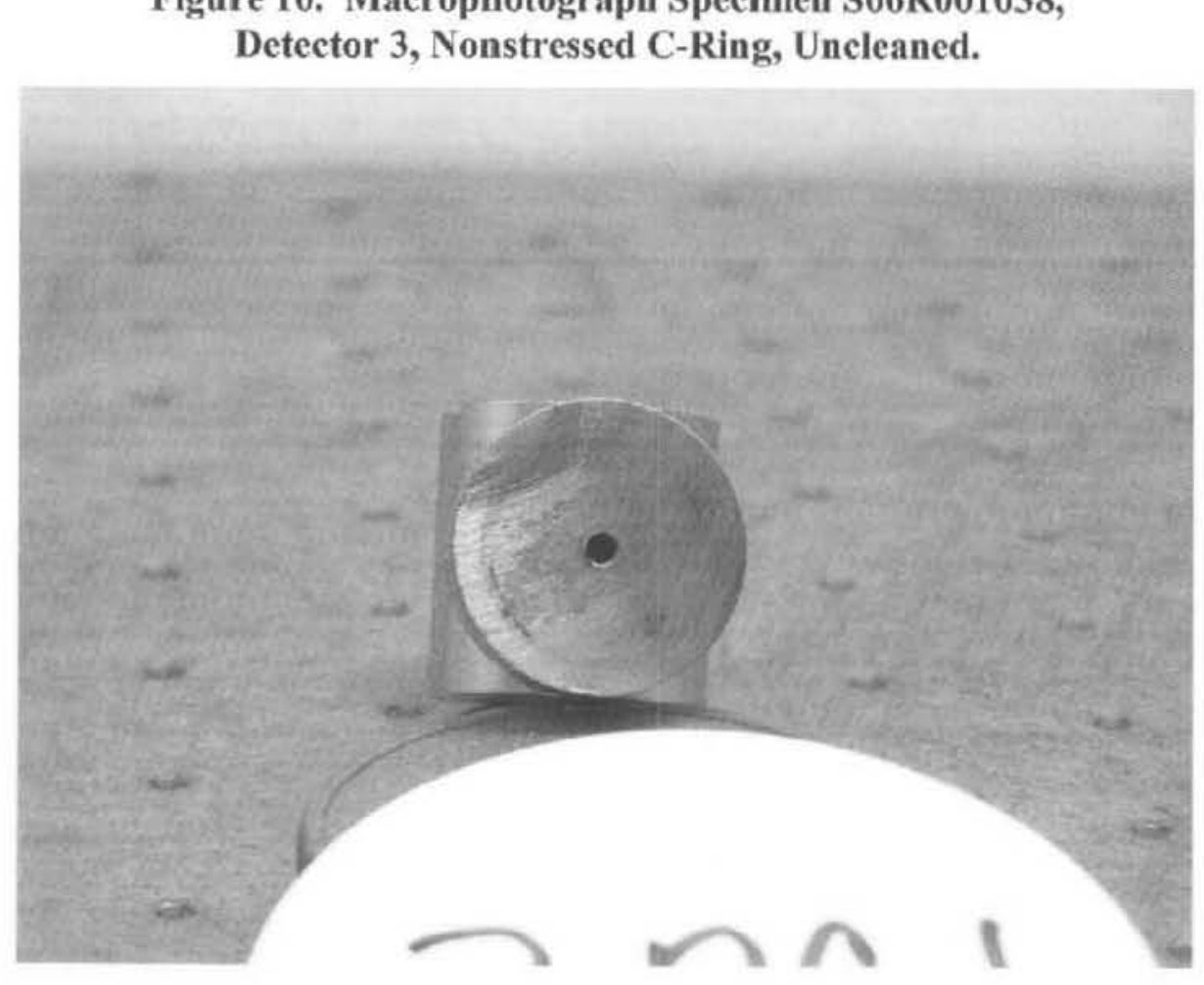



Detector 3, Stressed C-Ring, Uncleaned.

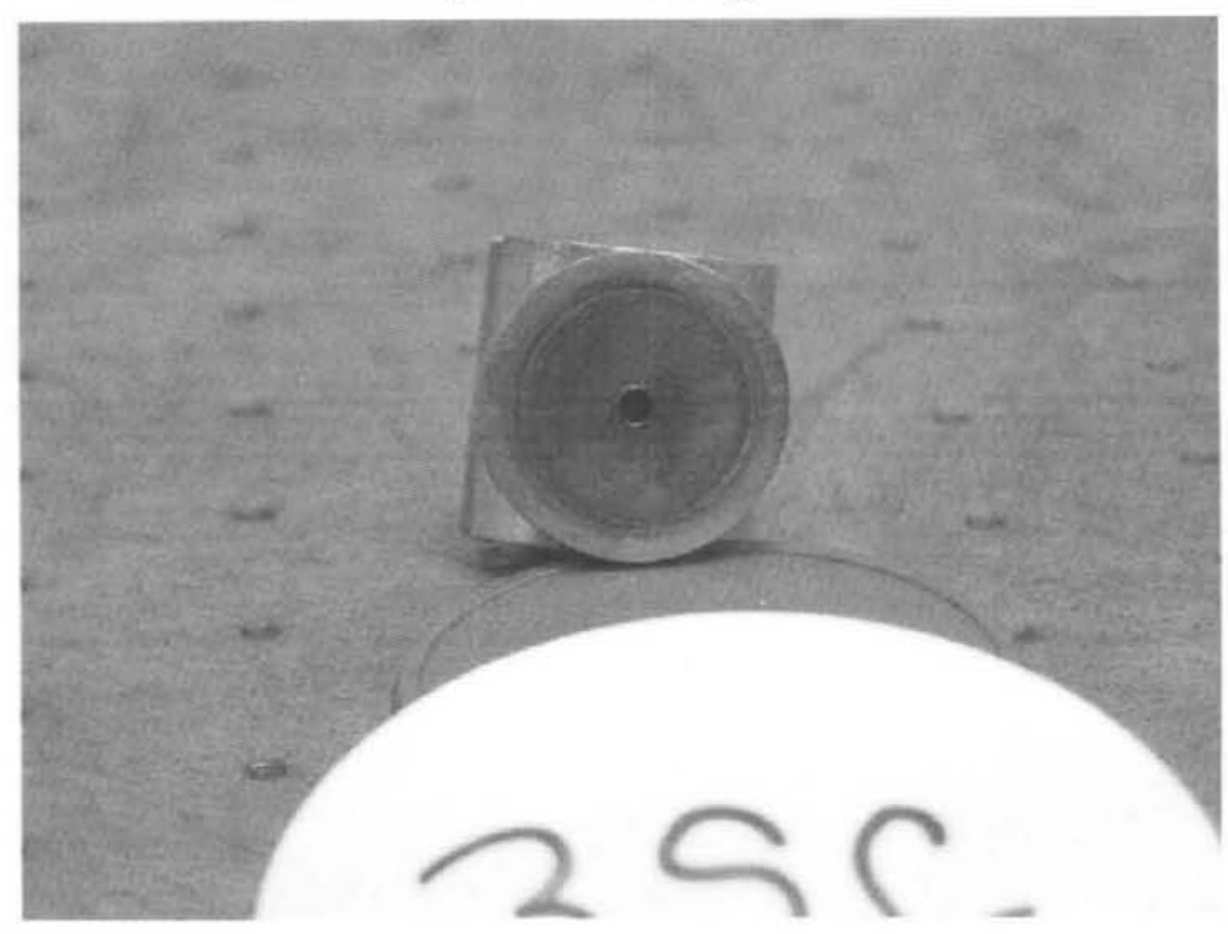

Figure 12. Macrophotograph Specimen S06R001042, Detector 4, Uncleaned.

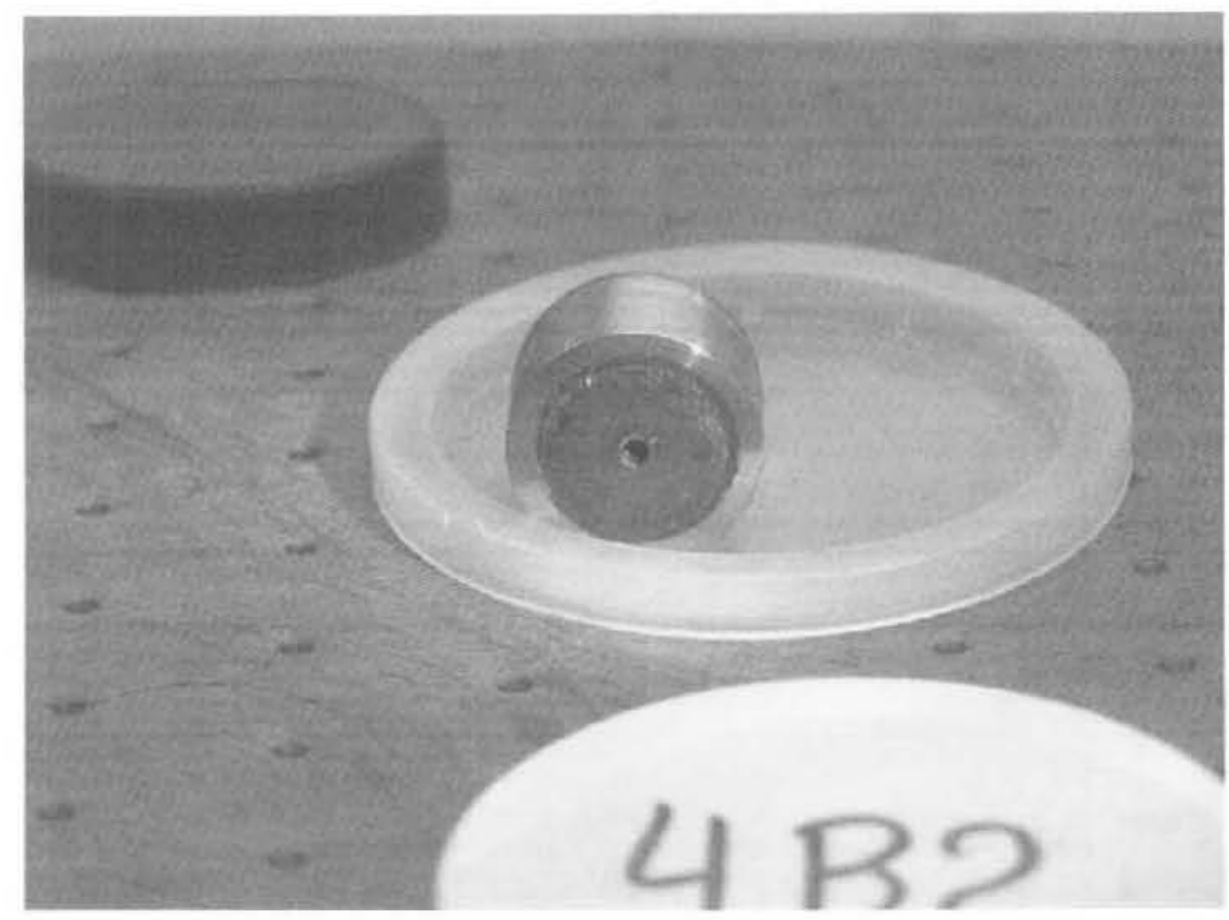

\section{Figure 11. Macrophotograph Specimen S06R001039,}




\section{Figure 13. Macrophotograph Specimen S06R001045, Detector 4, Stressed C-Ring, Uncleaned.}

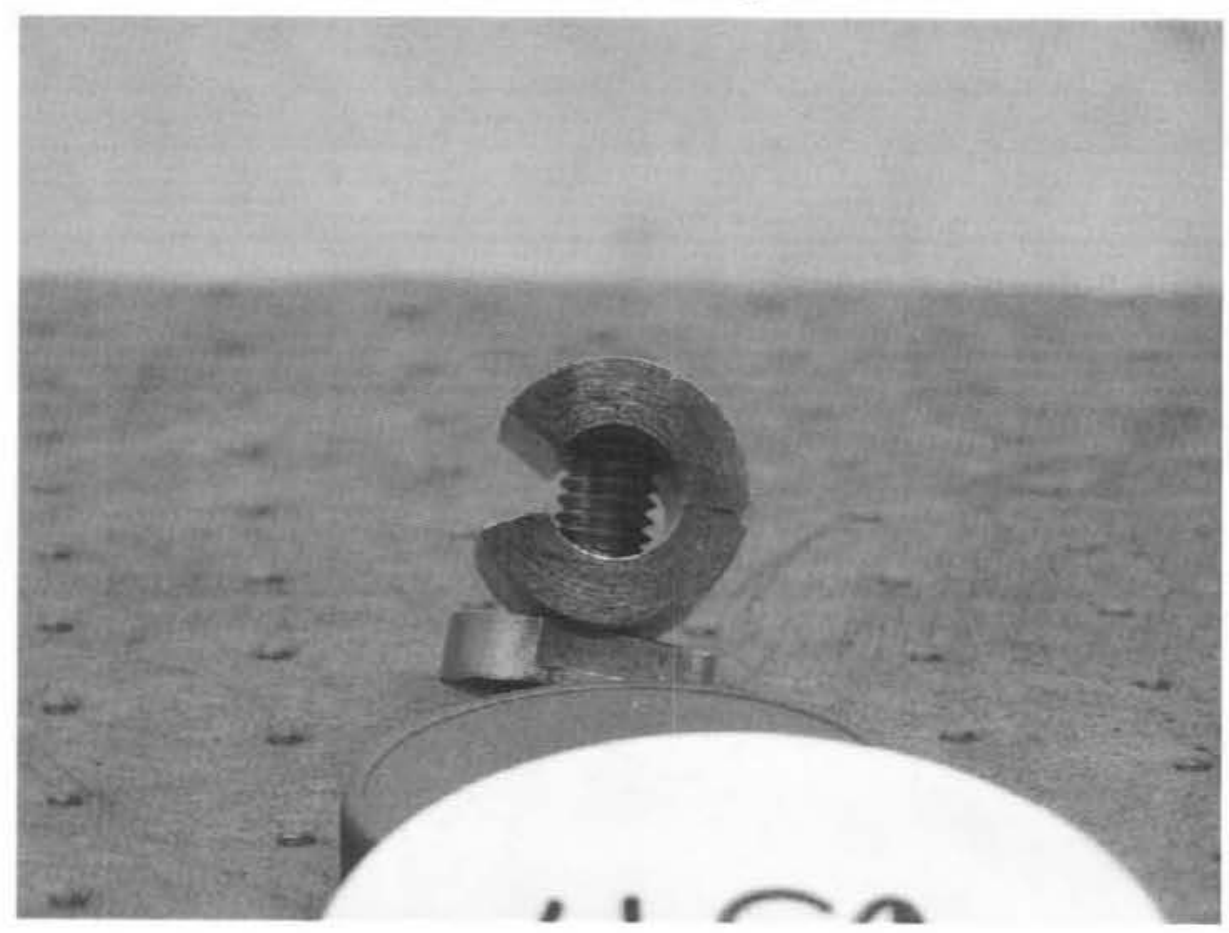

Specimen preparation followed the test procedures in 7S1 10-GAC-06-079 Reissue. Cleaning was performed with single or multiple washings with hot ammonium citrate in accordance with ASTM G46-94, Standard Guide for Examination and Evaluation of Pitting Corrosion. Table 2 compiles the weight loss data for the several washes necessary to achieve a minimal weight change after the initial wash $(<0.1 \%$ total weight loss) or between successive washes ( $<10.0 \%$ cumulative weight loss).

Cleaned specimens were examined by OM and SEM. Several features of note were documented before any further preparation. Specimen S06R001028, a nonstressed C-ring from detector 1 (in the vapor space) showed significant corrosion where the threads of the bolt came through the top of the C-ring (Figure 14). After cleaning, SEM examination of that region revealed that only a portion of the corrosion-covered area had significant pitting (Figure 15). 
Table 2. Weight Loss during Ammonium Citrate Cleaning.

\begin{tabular}{|c|c|c|c|c|c|c|}
\hline $\begin{array}{c}\text { Specimen } \\
\text { Identification } \\
1 \\
\end{array}$ & $\begin{array}{c}\text { Laboratory } \\
\text { Sample } \\
\text { Number } \\
2 \\
\end{array}$ & $\begin{array}{c}\begin{array}{c}\text { Weight } \\
\text { before }\end{array} \\
\text { Solution } \\
\text { Soak } \\
\text { (grams) } \\
3 \\
\end{array}$ & $\begin{array}{l}\text { Weight } \\
\text { after } \\
\text { Solution } \\
\text { Soak } \\
\text { (grams) } \\
4 \\
\end{array}$ & $\begin{array}{c}\text { Cumulative } \\
\text { Weight } \\
\text { Loss } \\
\text { (grams) } \\
5 \\
\end{array}$ & $\begin{array}{l}\text { Cleaning } \\
\text { Number }\end{array}$ & $\begin{array}{c}\text { Weight } \\
\text { Delta } \%{ }^{2} \\
6 \\
\end{array}$ \\
\hline Detector $1 \mathrm{~B} 2(\mathrm{Z})$ & S06R001024 & 82.345 & 82.270 & 0.075 & 1 & 0.091 \\
\hline Detector 1 B2 (Z) & S06R001024 & 82.270 & 82.242 & 0.103 & 2 & 27.184 \\
\hline Detector $182(\mathrm{Z})$ & SO6R001024 & 82.242 & 82.209 & 0.136 & 3 & 24.265 \\
\hline Detector $1 \mathrm{~B} 2(\mathrm{l})$ & S06R001024 & 82.209 & 82.214 & 0.131 & 4 & {$[3.81]$} \\
\hline Detector $1 \mathrm{SC}(\mathrm{W})$ & S06R001027 & 86.895 & 86.683 & 0.212 & 1 & 0.243 \\
\hline Detector $1 \mathrm{SC}(\mathrm{W})$ & S06R001027 & 86.683 & 86.608 & 0.287 & 2 & 26.132 \\
\hline Detector $1 \mathrm{SC}(\mathrm{W})$ & S06R001027 & 86.608 & 86.510 & 0.385 & 3 & 25.454 \\
\hline Detector $1 \mathrm{SC}(\mathrm{W})$ & S06R00102? & $86 . \overline{510}$ & 86.515 & 0.380 & 4 & {$[1.31]$} \\
\hline Detcctor $1 \mathrm{RC} 2(\mathrm{~V})$ & S06R001028 & 87.427 & 87.197 & 0.230 & I & 0.263 \\
\hline Detector $1 \mathrm{RC} 2(\mathrm{~V})$ & S06R001028 & 87.197 & 87.072 & 0.355 & 2 & 35.211 \\
\hline Detectur $1 \mathrm{RC} 2$ (V) & S06R001028 & 87.072 & 87.019 & 0.408 & 3 & 12.990 \\
\hline Detector $1 \mathrm{RC} 2(\mathrm{~V})$ & SO6R001028 & 87.019 & 86.946 & 0.481 & 4 & 15.177 \\
\hline Detector I RC2 (V) & S06R001028 & $86 . \overline{946}$ & 86.943 & 0.484 & 5 & 0.620 \\
\hline Detector $2 \mathrm{SC}(\mathrm{P})$ & S06R001033 & 86.684 & 86.661 & 0.023 & 1 & 0.027 \\
\hline Detector 3 B2 (L) & S06R001036 & 82.512 & 82.511 & 0.001 & I & 0.001 \\
\hline Detector $3 \mathrm{SC}(\mathrm{H})$ & SO6R001039 & 88.426 & 88.411 & 0.015 & 1 & 0.017 \\
\hline Detector $4 \mathrm{~B} 2$ (E) & S06R001042 & 82.260 & 82.247 & 0.013 & 1 & 0.016 \\
\hline Detector $4 \mathrm{SC}(\mathrm{B})$ & SO6R001035 & 87.499 & 87.483 & 0.016 & I & 0.018 \\
\hline Centering Post & Blank & $4 \overline{7.798}$ & 47.770 & 0.028 & 1 & $0 . \overline{059}$ \\
\hline Centering Post & Blank & 47.770 & 47.766 & 0.032 & 2 & 12.5 \\
\hline
\end{tabular}

${ }^{a}$ Weight delta $\%$ is the weight loss [gain] as a percentage of the total sample for the first cleaning. For successive cleaning, it is the percentage weight loss as a percentage of the cumulative weight loss from all cleanings. 
Figure 14. Macrophotograph of the Top of Vapor Space Nonstressed C-Ring S06R001028.

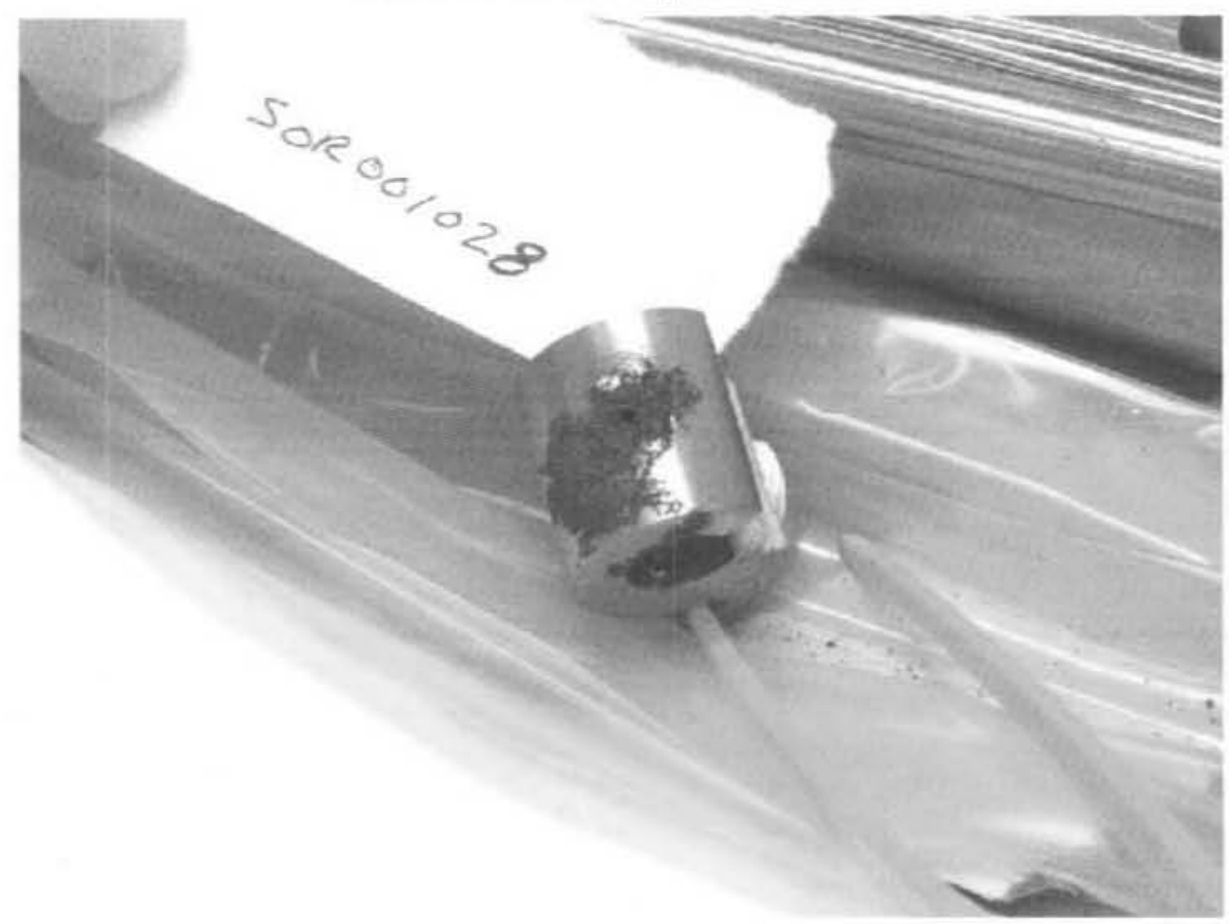

Figure 15. SEM Mierophotograph of the Top of Vapor Space Nonstressed C-Ring S06R001028.

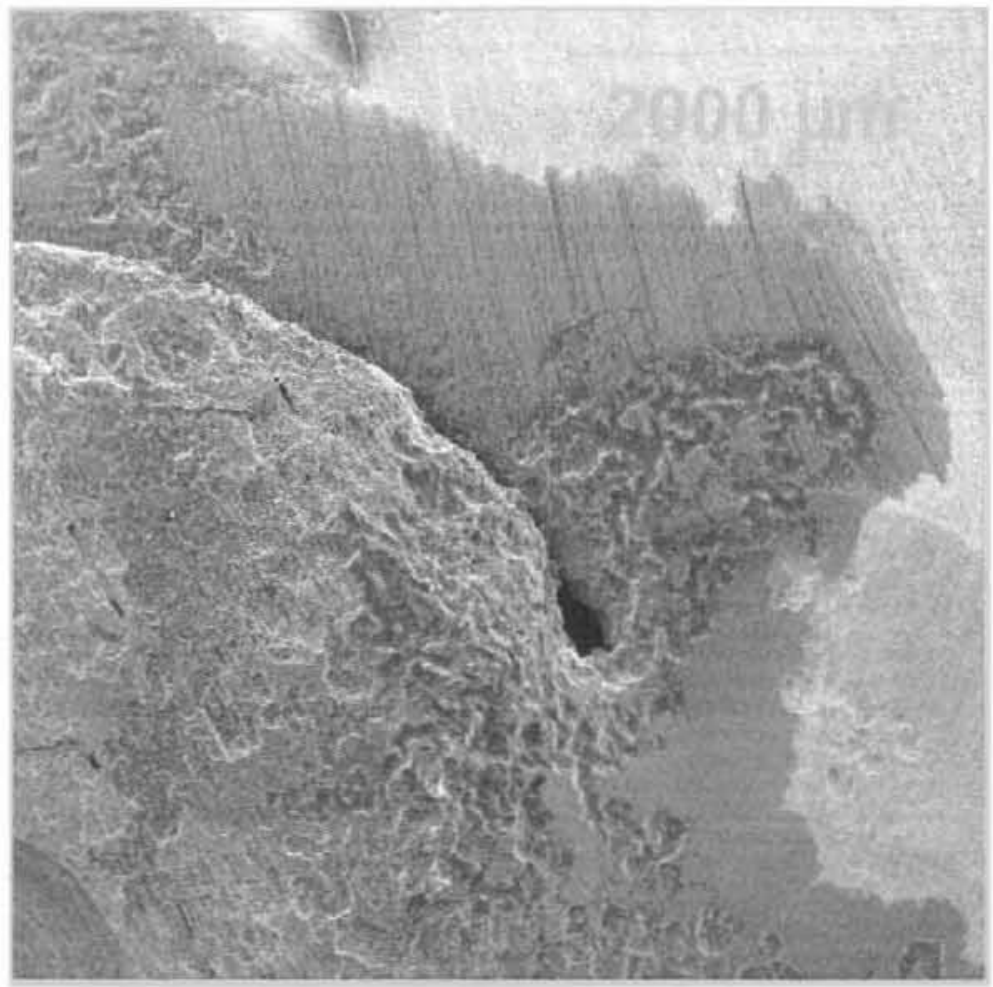


Following cleaning, the selected specimens were prepared for examination in several ways. Cross sections of the specimens were sliced using a low-speed saw with a diamond-impregnated blade. The stressed C-rings were prepared for examination of the stress-cracked section by slicing a thin section of the C-ring. The thin (about 4-mm thick) portion of the stressed C-rings was submerged in liquid nitrogen (LN2) and then struck lightly, on edge, with a mallet, to complete the fracture into two pieces. These two pieces were mounted side-by-side for $\mathrm{OM}$ and SEM examination of the fractured surfaces. Figure 16 is an SEM photomicrograph of one of the fractured surfaces from S06R001027, the vapor space stressed C-ring. All measurements in Figure 16 start at the top where the v-notch ends and the pre-crack begins. The entire length of the pre-crack after recovery and preparation is $3720 \mu \mathrm{m}$. The region from $3720 \mu \mathrm{m}$ to $5230 \mu \mathrm{m}$ is the LN2 fractured surface. Figure 17 through Figure 19 show example SEM photomicrographs of the three stressed C-ring LN2 fractured surfaces. In these three figures, the right-hand image is a magnified view of the area in the yellow box of the left hand image and the LN2 fractured portion is toward the bottom of the images. These right-hand images are all taken from the region where the pre-crack ends and the LN2 fracture surface begins.

Figure 16. SEM Photomicrograph of the Face of Pre-stressed Crack, Specimen S06R001027.

(V-notch is toward the top and the LN2 cracked area is toward bottom.)

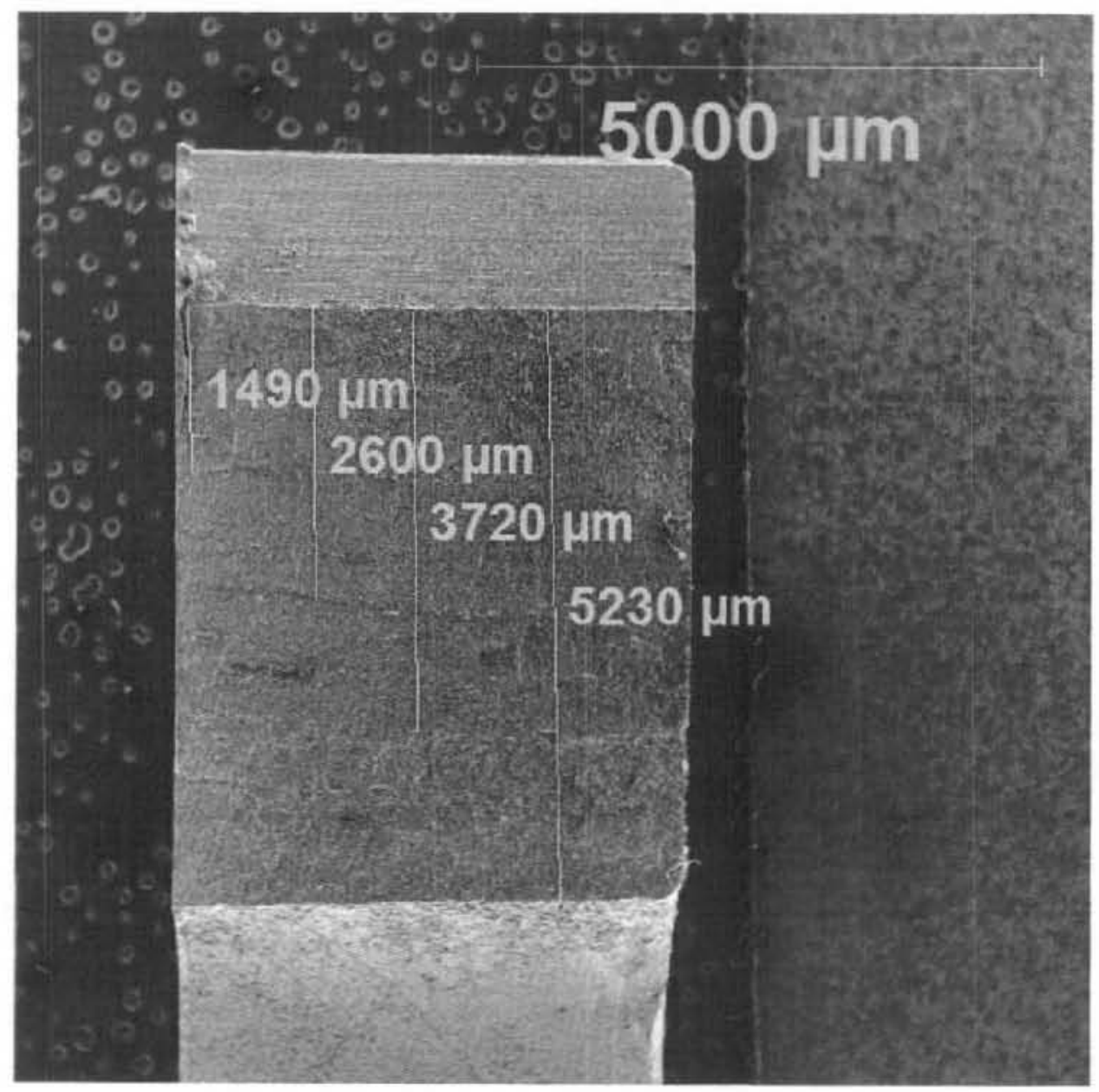


Figure 17. SEM Photomicrograph of the Face of Pre-stressed Crack, Specimen S06R001027.

(Image to the right is a blow-up of the area within the box in the left-hand image.)
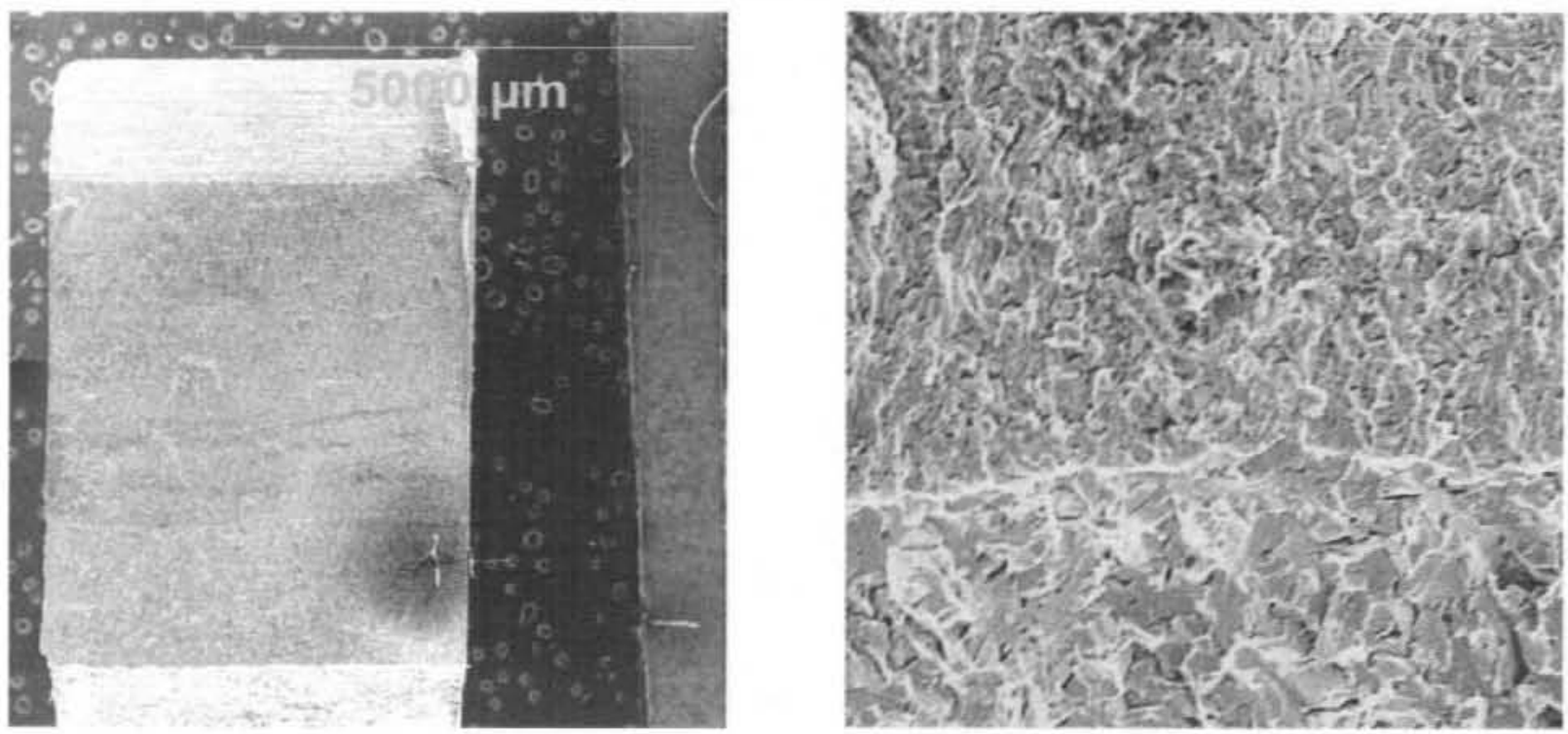

Figure 18. SEM Photomicrograph of the Face of Pre-stressed Crack, Specimen S06R001033.

(Image to the right is a blow-up of the area within the box in the left-hand image.)
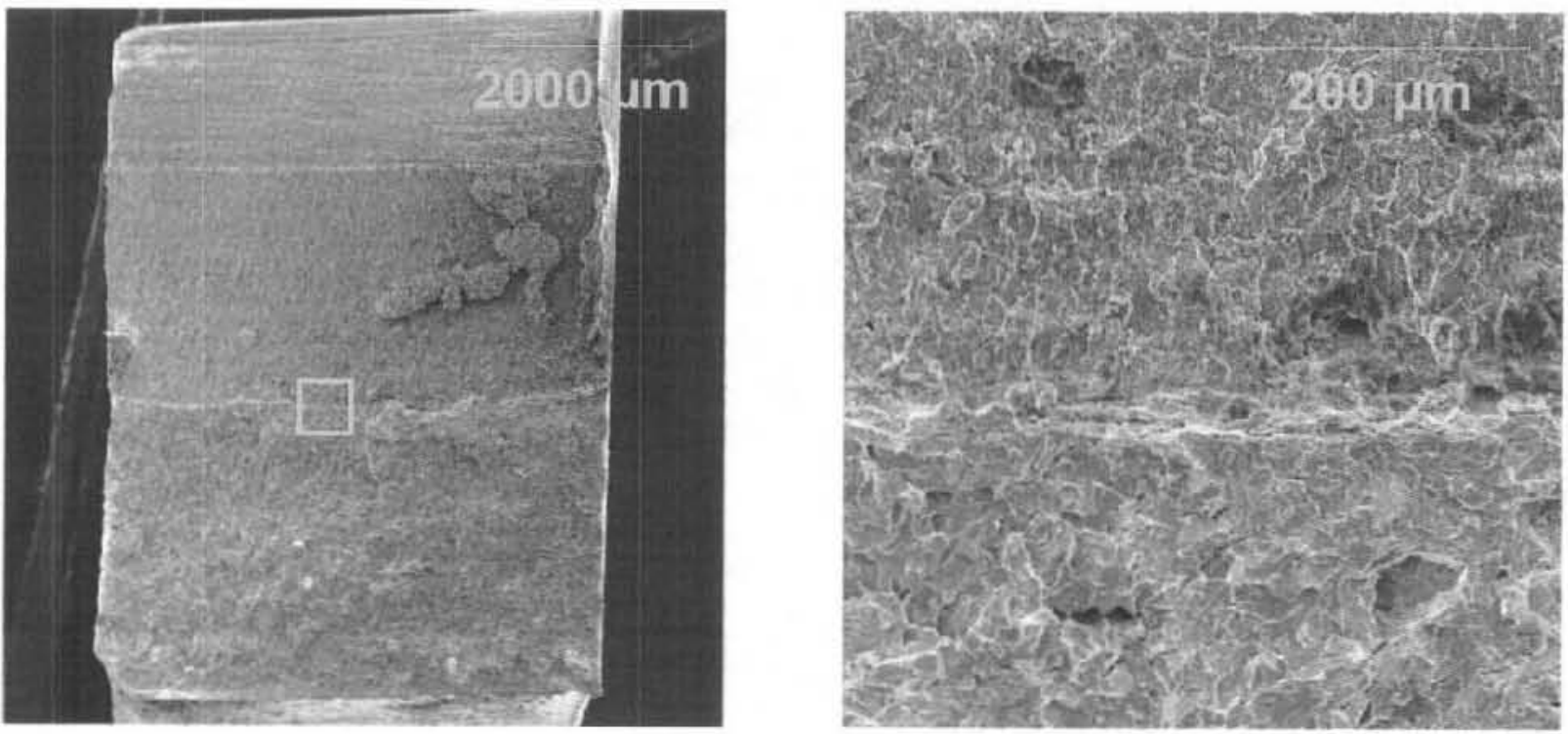
Figure 19. SEM Photomicrograph of the Face of Pre-stressed Crack, Specimen S06R001045.

(Image to the right is a blow-up of the area within the box in the left-hand image.)
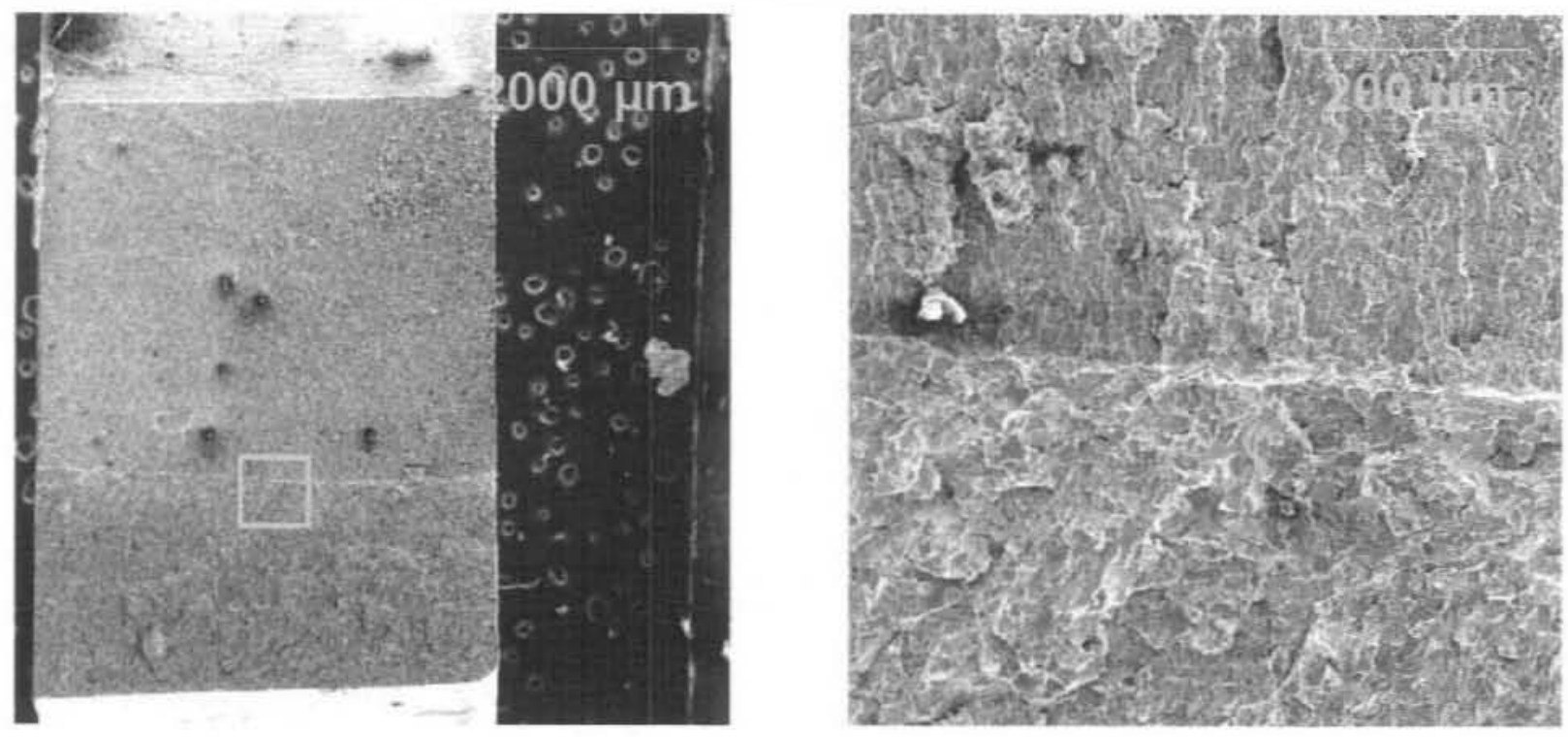

The larger cut sections of two of the pre-stressed C-rings were polished with successively finer polishing grits with a 10- $\mu \mathrm{m}$ final polish. The cut and polished surfaces were then etched with $3 \%$ nitric acid in methanol (ASTM E-407-99, Standard Practice for Microetching Metals and Alloys). The polished and etched surfaces of the pre-cracks were again examined by $\mathrm{OM}$ and SEM. Figure 20 and Figure 21 show SEM photomicrographs of the polished and etched surfaces at the tips of each of the pre-cracks in the two stressed C-rings that were examined.

Finally, a number of specimens were examined for corrosion pit size, depth, and shape. These samples were prepared by cutting cross-sections using the low-speed saw and polishing the surfaces to 600 grit. The resulting specimens were examined by OM and SEM. Representative images of the largest pits observed in these samples are shown in Figure 22 through Figure 26. 
Figure 20. SEM Photomicrograph of the Polished and Etched Surface of Pre-stressed Crack, Specimen S06R001027.

(Image to the right is a blow-up of the area within the box in the left-hand image.)
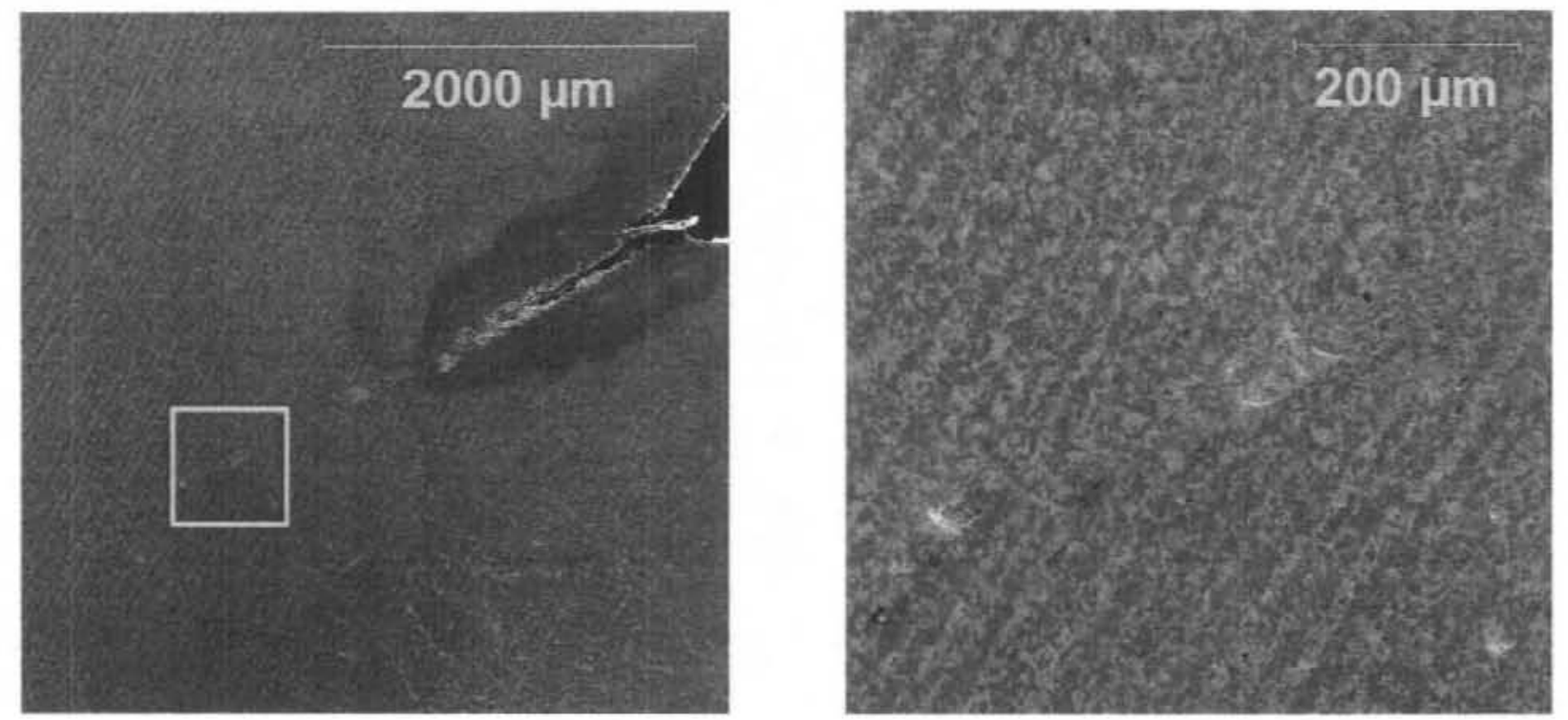

Figure 21. SEM Photomicrograph of the Polished and Etched Surface of Pre-stressed Crack, Specimen S06R001045.

(Image to the right is a blow-up of the area within the box in the left-hand image.)
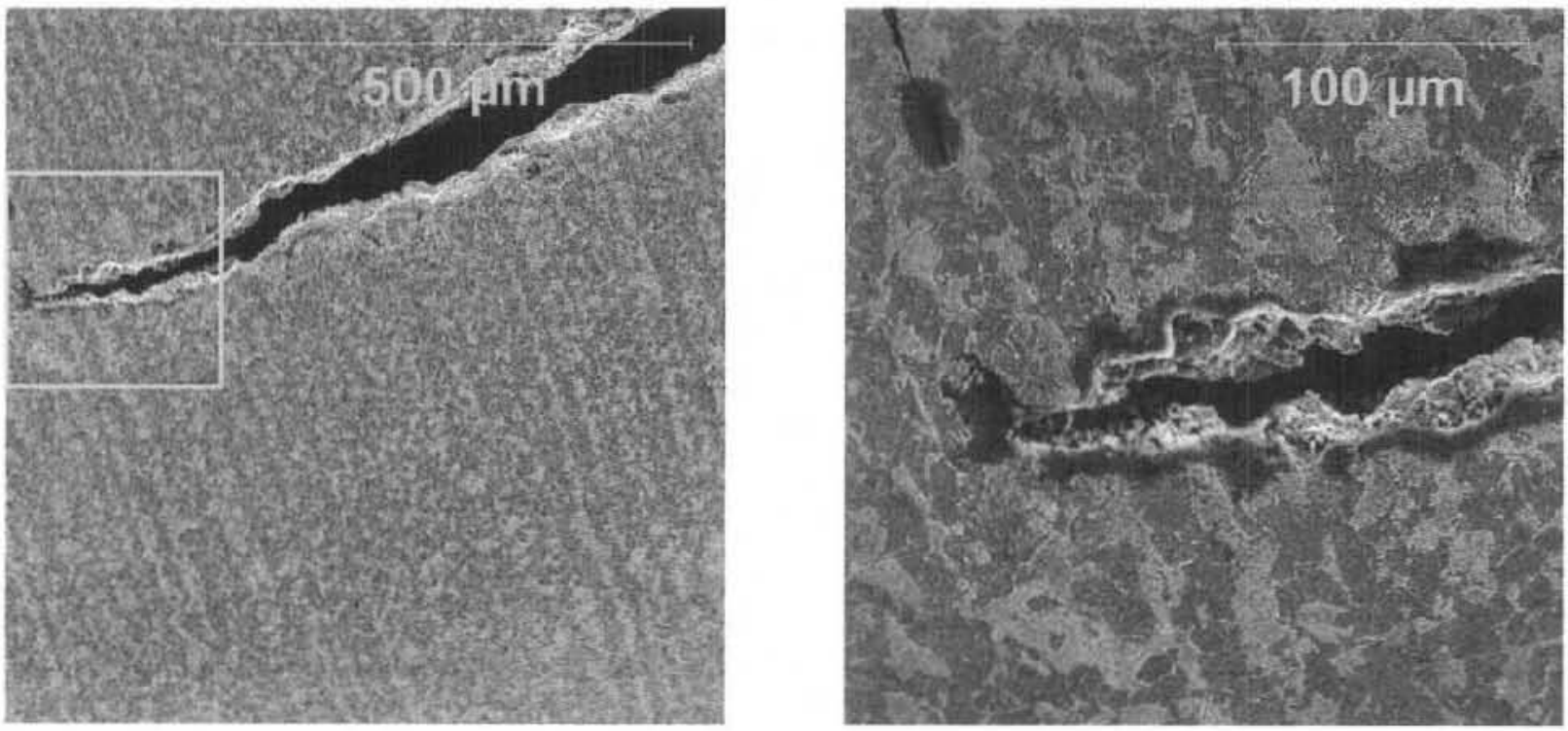


$$
\text { RPP-RPT-32425, Rev. } 0
$$

Figure 22. SEM Image of Pitted Area on Outer Surface of Sample S06R001024.
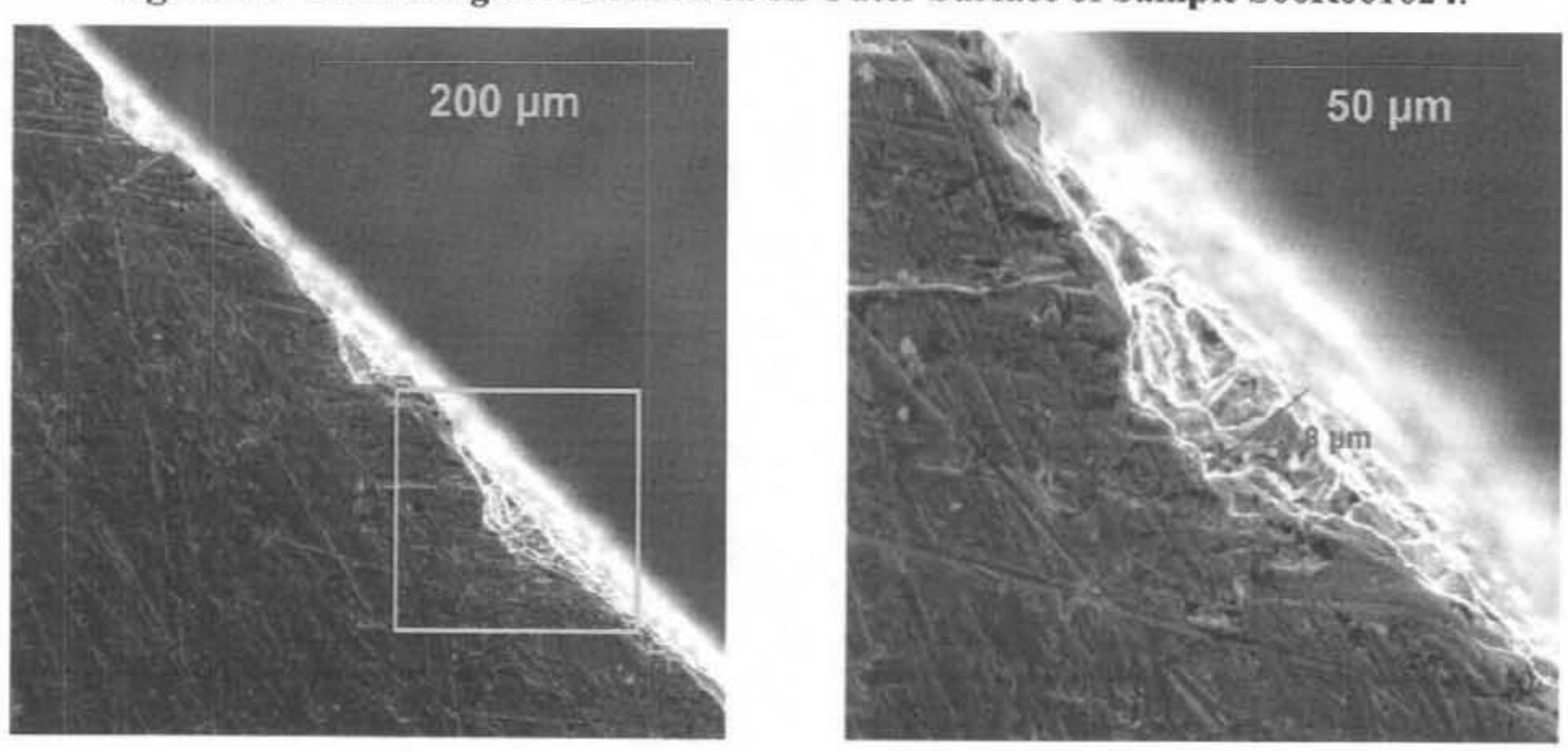

Figure 23. SEM Image of Pitted Area Near Crevice Between C-Ring and Bolt Head on Sample S06R001027.
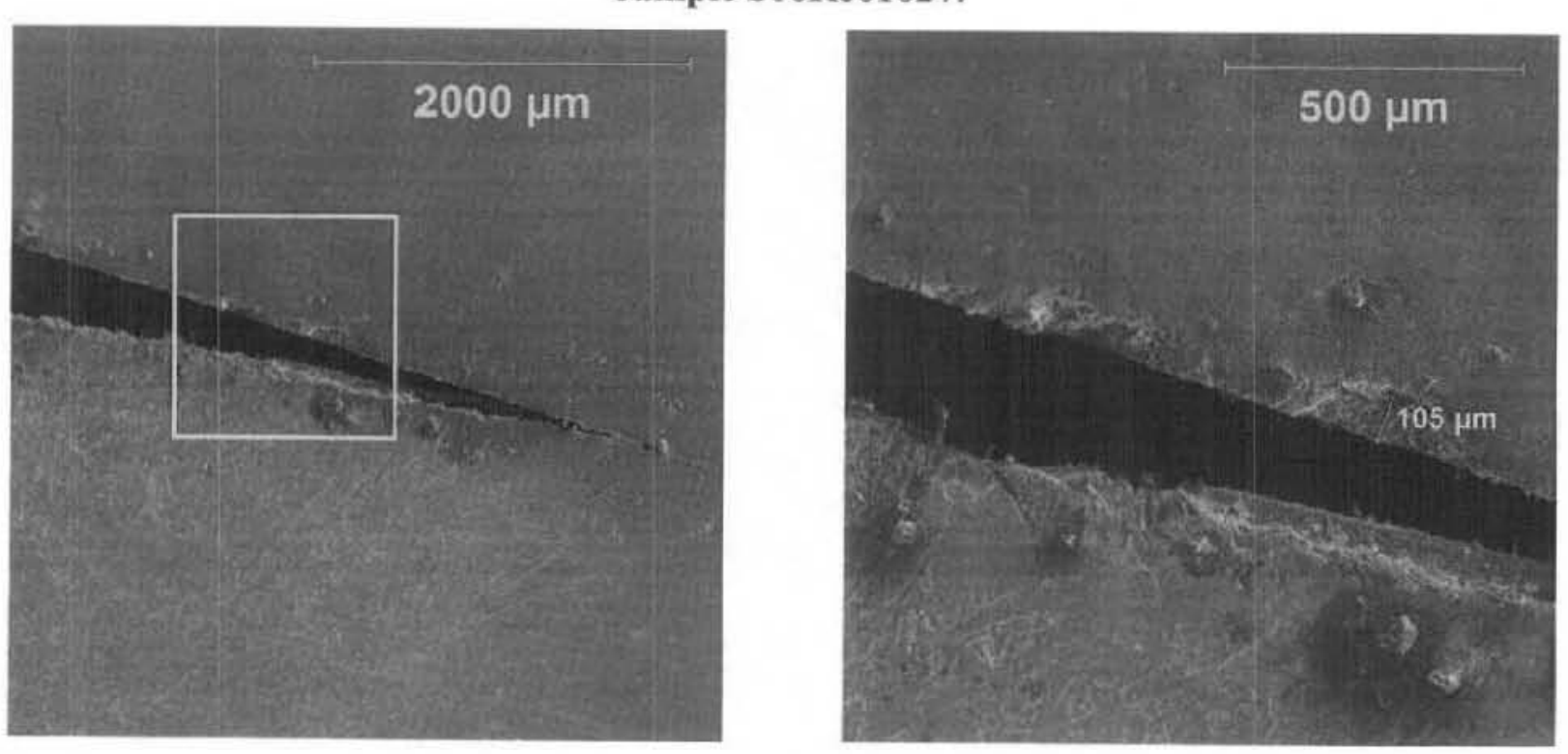
Figure 24. SEM Image of Pitted Area from End of Bolt on Sample S06R001028.
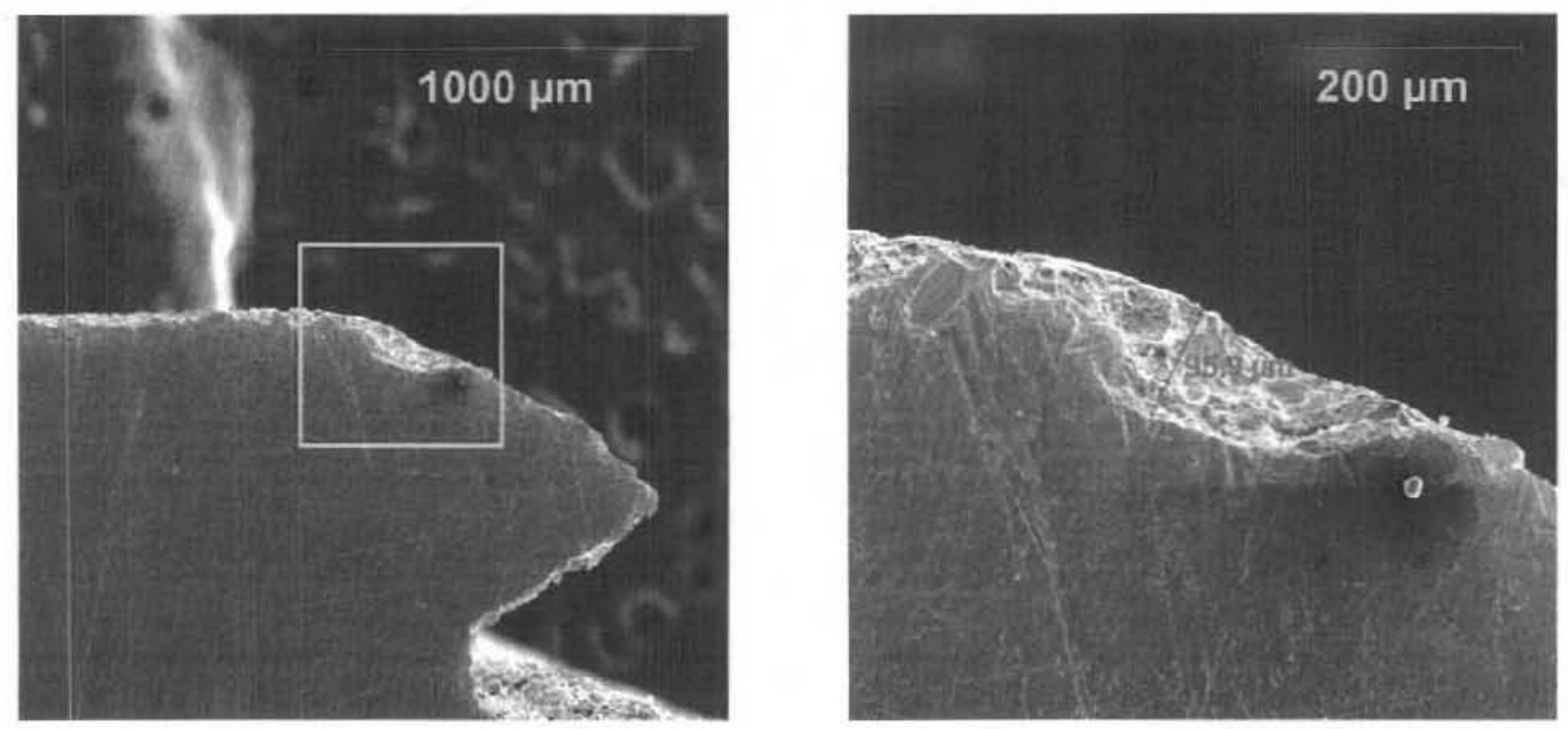

Figure 25. SEM Image of Pitted Area on Base of Sample S06R001042.
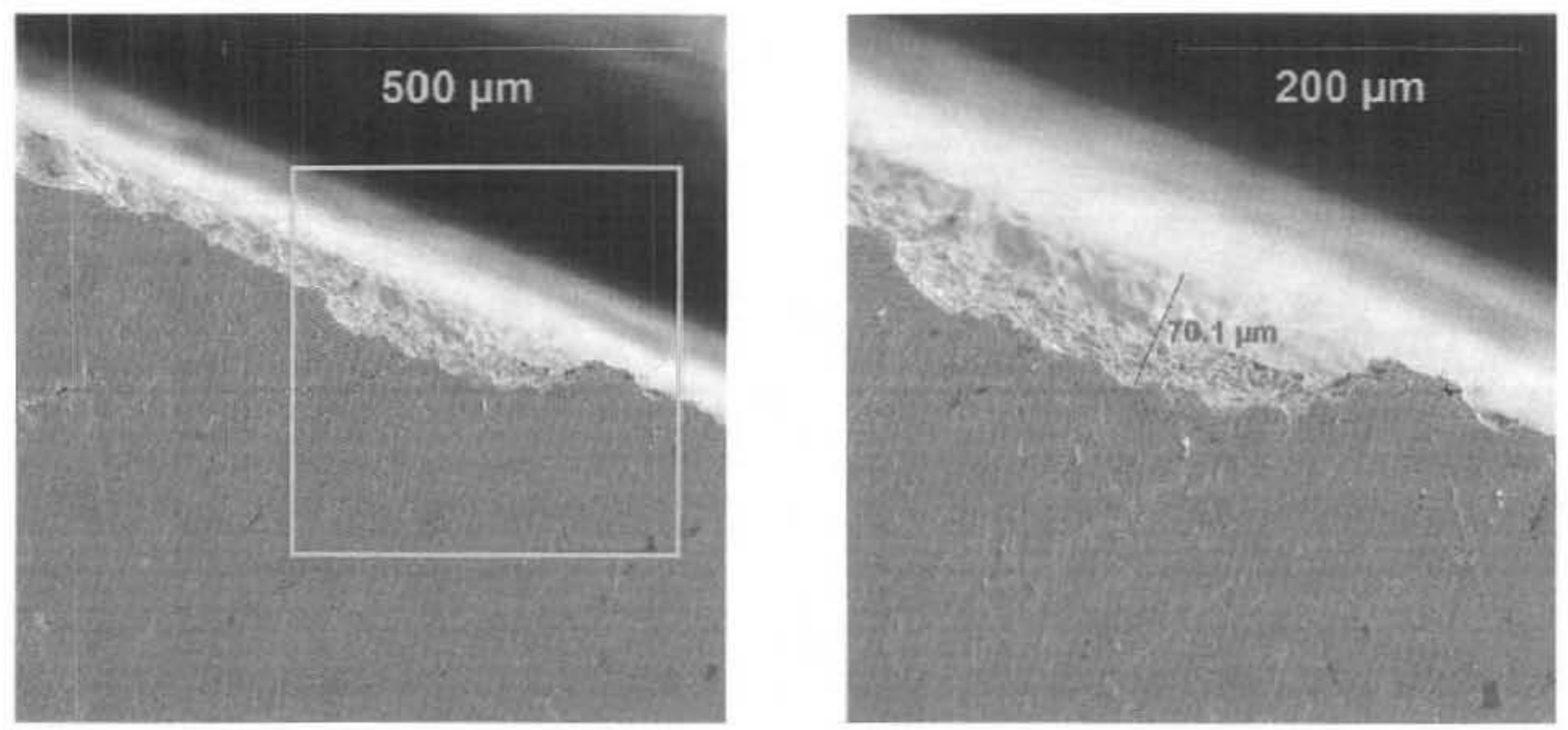
Figure 26. SEM Image of Pitted Area on Sample S06R001045.
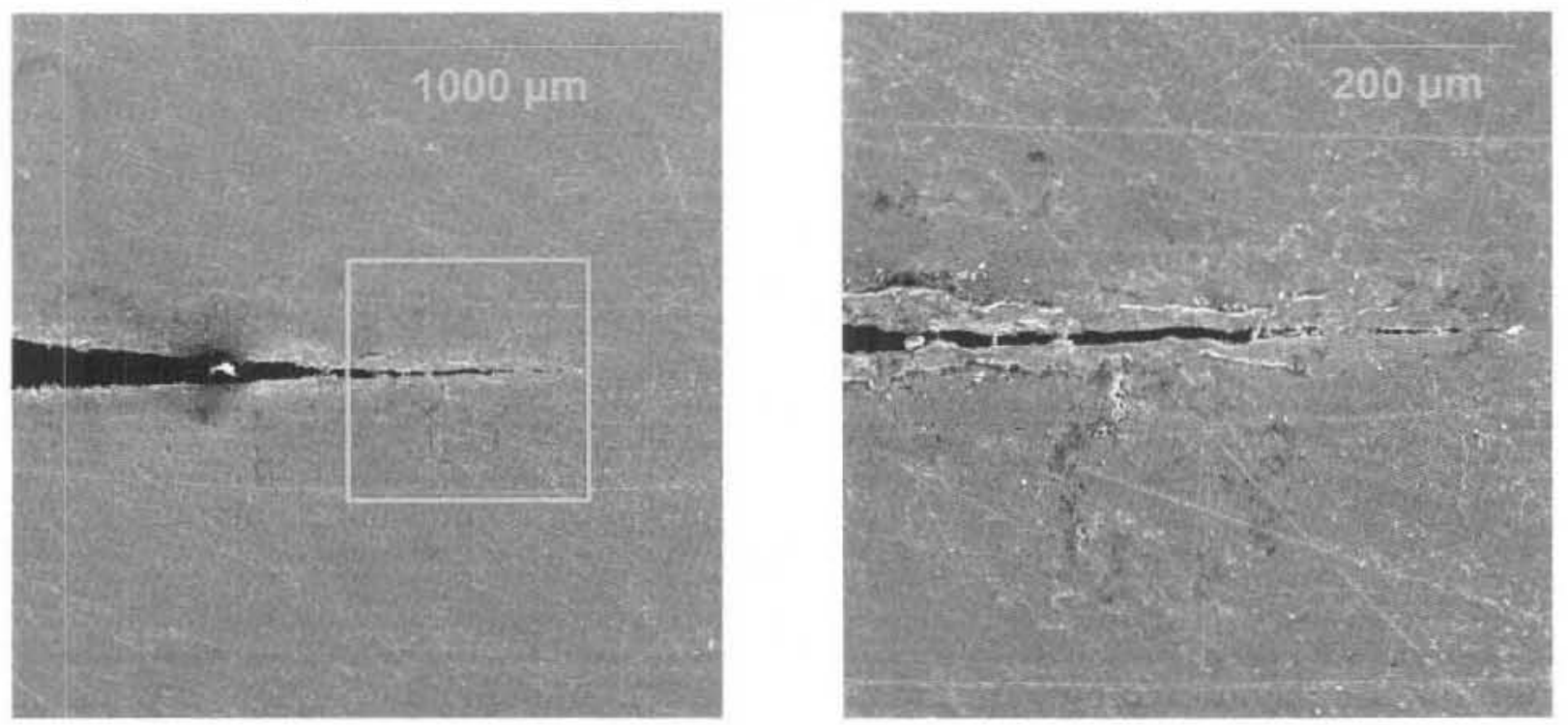

\section{DISCUSSION}

The AN-107 electrochemical noise probe was removed from AN-107 on August 10, 2006, cut into four sections, wrapped, placed in a 55-gallon drum and delivered to the 222-S Laboratory on August 17,2006 . The 222-S Laboratory unpackaged the sections of the AN-107 electrochemical noise probe and examined the material for evidence of corrosion.

The electrochemical noise probe consisted of four detector sections, each containing three $\mathrm{C}$-ring specimens and three bullets. Detector 1 was deployed in the head space or vapor space of the tank. Detector 2 and Detector 3 were submerged in the supernate liquid. Detector 4 was imbedded in the saltcake waste. One of the three $\mathrm{C}$-ring specimens on each detector was pre-stressed to investigate the potential for stress corrosion cracking in the tank waste.

Detcetor 1 was disassembled in a fume hood and all six specimens were removed and examined during rinsing and transfer to the dessicator. During this process, it was noted that the prestressed C-ring S06R001027 had a noticeable crack extending from the tip of the $v$-notch (Figure 7). In addition, significant patches of corrosion were seen on several of the other specimens on detector 1 . Of note were a patch of surface corrosion on bullets S06R001024 and S06R001025 (Figure 1 and Figure 6) and a large patch of corrosion on the top of nonstressed C-ring S06R001028 where the bolt extended through the C-ring (Figure 14). It was also noted that all of the specimens removed from detector 1 had significant corrosion on the underside, beneath, and behind the region that was protected by the $\mathrm{O}$-ring (Figure 27). 
RPP-RPT-32425, Rev. 0

Figure 27. Bullet Sample S06R001023. (Bottom region, behind O-ring.)

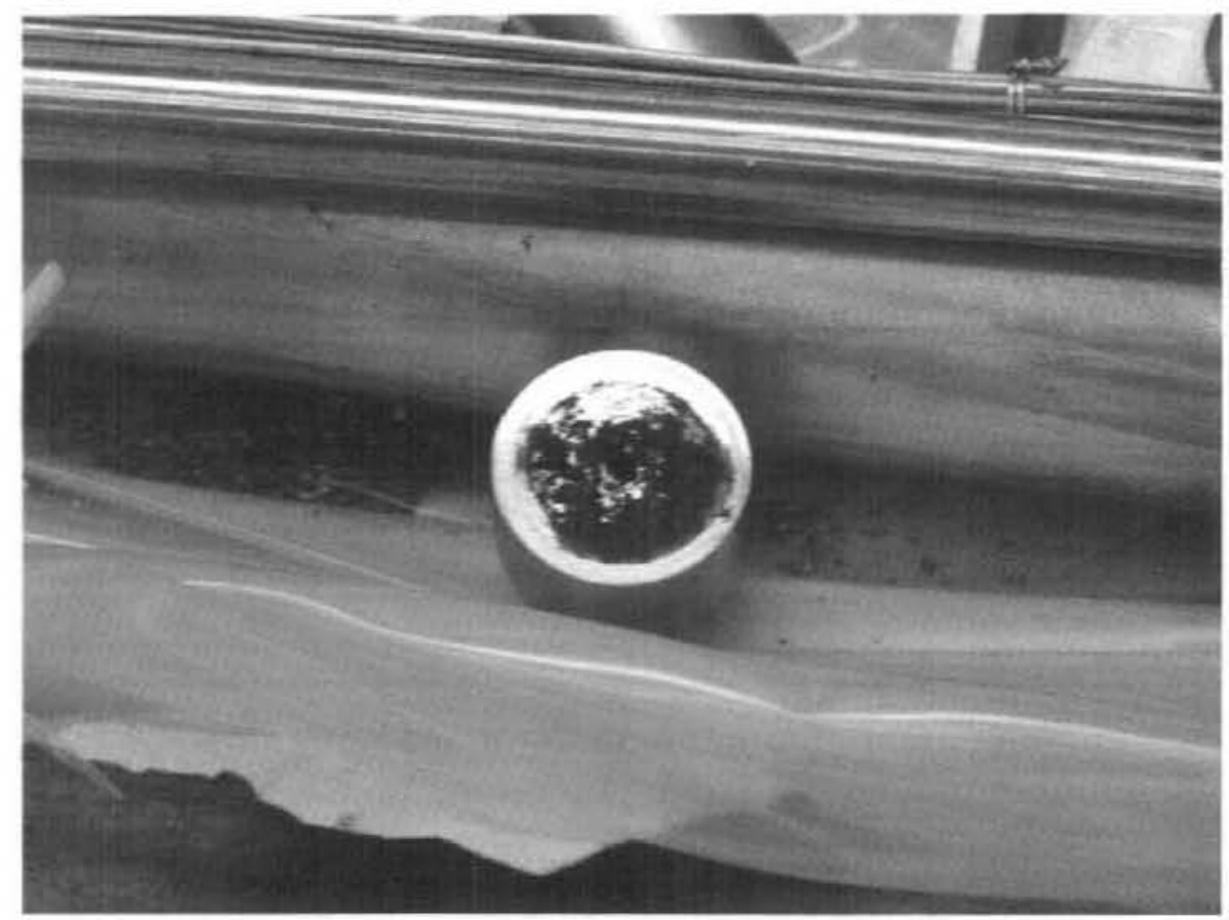

During discussions with Glenn Edgemon of ARES Corporation about observations made during the Detector 1 breakdown, it was learned that the stressed C-rings were not stressed to just below the point of cracking, as specified in ASTM G38-01, Standard Practice for Making and Using C-Ring Stress Corrosion Test Specimens. Rather, they were stressed beyond failure to "precrack" the stressed C-rings. Therefore, the observation of cracks in the v-notch of specimen S06R001027 was to be expected. Unfortunately, no pictures of the pre-cracked specimens were available. Therefore, the extent to which cracking occurred in the tank becomes more difficult to determine and the physical evidence from analysis of the $\mathrm{C}$-rings provides the only basis, other than analysis of the electrochemical signals, for determining if stress corrosion cracking has occurred. Edgemon also indicated (personal communication) that the observation of corrosion product beneath the Detector 1 specimens and behind the O-rings was not unexpected since the electrical signal from Detector 1 failed early during deployment.

Disassembly of the remaining detectors revealed that the other three pre-stressed C-rings were also cracked in the v-notch, as was now expected. It also showed that the submerged detector section specimens were coated with significantly less corrosion product. Corrosion apparently proceeded at a greater rate in the vapor space of the tank.

During disassembly of all four detectors, the sections were photographed and eight specimens were selected for microscopic evaluation of corrosion. These were the four pre-stressed C-rings (all of which showed evidence of pre-cracking) and four additional samples (see Table 1). The additional samples were, in general, selected because they showed some evidence for surface pitting or crevice corrosion. 
The cleaning of these specimens in accordance with ASTM G1-90, Standard Practice for Preparing, Cleaning, and Evaluating Corrosion Test Specimens, serves two purposes. It removes corrosion to expose the metal surface to examine for the extent and type of corrosive attack, and it provides weight loss data for the estimation of corrosion rates. None of the C-rings or bullet specimens were preweighed prior to deployment in the tank; therefore, there is no basis for conversion of weight loss data from specimen preparation to a corrosion rate. The data obtaincd during clcaning confirmed more corrosion product had built up on the vapor space specimens than on the specimens from the other three detectors (Table 2). Weight losses during cleaning for the three specimens in the vapor space were $0.131 \mathrm{~g}, 0.380 \mathrm{~g}$, and $0.484 \mathrm{~g}$. None of the weight losses during cleaning for the other five spocimens cxceeded $0.03 \mathrm{~g}$.

The specimens were cxamined for pitting corrosion, crevice corrosion, and stress corrosion cracking, as discussed in the ASM Handbook, Corrosion, Volume 13. Macrophotography, OM, and SEM were used to document the disassembly process and to determine the extent of corrosion on each of the eight spccimens.

No evidence of stress corrosion cracking was found in the stressed C-ring specimens. These examination results were reported in internal letter 7S110-GAC-06-087, "Results of the Examination of Electrochemical Noise Probe Specimens Removed from Tank 241-AN-107 August 10, 2006" (7S110-GAC-06-087). Minor pitting was evident on some surfaces. Crevice corrosion was the dominant type of corrosion observed.

Crevice corrosion was found at the top of the C-rings (where the threaded bolt met the C-ring), at the base of the $\mathrm{C}$-ring (where the bolt conlacted the $\mathrm{C}$-ring), and beneath the $\mathrm{C}$-ring and bullet specimens, where vapors and liquids penetrated behind the $\mathrm{O}$-ring seals and caused crevice corrosion between the detector elements and the $\mathrm{O}$-rings. Pitting corrosion was found in isolated patches on the surfaces of some of the C-rings and bullets. Both crevice and pitting corrosion were more noticeable on the specimens that had been suspended in the vapor space of the tank (Detector 1) but evidence of crevice and pitting corrosion was also present on some of the other specimens recovered from the detectors submerged in the waste.

Cleaned, cut, and polished sections revealed that pitting was not very wel1 developed in the corroded areas. Pit depths from the cross-sectioned specimens were always less than $100 \mu \mathrm{m}$. Polished and/or freeze-fractured surfaces of the stressed C-rings showed no evidence of stress corrosion cracking.

\section{REFERENCES}

7S110-GAC-06-079 Reissue, 2006, "Transmit the Tcst Procedure for the Examination of Electrochemical Noise Probe Specimens to be Removed from Tank 241-AN-107, August 2006" (internal letter from G. A. Cooke to K. G. Carothers, September 20), CH2M HILL Hanford Group, Inc., Richland, Washington. 
7S110-GAC-06-087, 2006, "Results of the Examination of Electrochemical Noise Probe Specimens Removed from Tank 241-AN-107 August 10, 2006" (internal letter from G. A. Cooke and J. B. Duncan to K. G. Carothers, September 27), CH2M HILL Hanford Group, Inc., Richland, Washington.

ASM Handbook, 1987, Volume 13, Corrosion, ASM Intemational, Materials Park, Ohio.

ASTM G1-90, 1990 (Reapproved 1999), Standard Practice for Preparing, Cleaning, and Evaluating Corrosion Test Specimens, ASTM International, West Conshohocken, Pennsylvania.

ASTM G38-01, 2001, Standard Practice for Making and Using C-Ring Stress-Corrosion Test Specimens, ASTM International, West Conshohocken, Pennsylvania.

ASTM G46-94, 1994 (Reapproved 1999), Standard Guide for Examination and Evaluation of Pitting Corrosion, ASTM International, West Conshohocken, Pcnnsylvania.

ASTM E-407-99, 1999, Standard Practice for Microetching Metals and Alloys, ASTM International, West Conshohocken, Pennsylvania. 
RPP-RPT-32425, Rev. 0

APPENDIX A

IMAGE NAMES AND LOCATIONS FROM THE EXAMINATION OF 241-AN-107 ELECTROCHEMICAL NOISE PROBE SPECIMENS

NOTE: Images located on the Hantord Chardocs share drive in the directory; AN107 Corrosion Probe/Final Report 
RPP-RPT-32425, Rev. 0

\section{Chardocs: LAN107 Corrosion ProbelFinal Report \ Detector Breakdown!}

Subdirectory Detector IISeptember Breakdownไ

AN107 Segment 1 028.jpg

AN107 Segment 1 029.jpg

AN107 Segment 1 030.jpg

AN107 Segment 1 031.jpg

AN107 Segmont 1 032.jpg

AN107 Segment 1 033.jpg

AN107 Segment 1 034.jpg

AN107 Segment 1 035.jpg

AN107 Segment 1 036.jpg

AN107 Segment 1037.jpg

AN107 Segment 1 038.jpg

AN107 Segment 1 039.jpg

AN107 Segment 1 040.jpg

AN107 Segment 1 041.jpg

AN107 Segment 1 042.jpg

AN107 Segment 1 043.jpg

AN1 07 Segment $1044 . j p g$

AN107 Segment $1045 . j p g$

AN107 Segment 1 046.jpg

AN107 Segment 1 047.jpg

AN107 Segment 1 048.jpg

AN107 Segment 1 049.jpg

AN107 Segment 1 050.jpg

AN107 Segment 1 051.jpg

AN107 Segment 1 052.jpg

AN107 Segment 1 053.jpg

AN107 Segment 1 054.jpg

Subdirectory Detector 2\December Breakdown

Section2B1HC1.JPG

Section2B1HC2.JPG

Section2B2HC1.JPG

Section2B2HC2.JPG

Section2B3HCl.JPG

Section2B3HC.2.JPG

Section2C1HC1.JPG

Section2C1HC2.JPG

Section2C3HCL.JPG

Section2C3HC2.JPG

Tree2-2.JPG

Tree2-3.JPG

Trec2-4.JPG 
RPP-RPT-32425, Rev. 0

Tree2-5.JPG

Tree2-6.JPG

Tree2-7.JPG

\section{Subdirectory Detector 2\September Breakdown \} Processed

Segment 2 Samplc tag.jpg

Segment 2-1.jpg

\section{Ravy}

AN107 Segment 3 and 4 006.jpg

AN107 Segment 3 and 4 007.jpg

AN107 Segment 3 and 4 008.jpg

AN107 Segment 3 and 4 009.jpg

AN107 Segment 3 and 4 010.jpg

AN107 Segment 3 and 4 011.jpg

AN107 Segment 3 and 4 012.jpg

AN107 Segment 3 and 4 013.jpg

AN107 Segment 3 and 4 014.jpg

AN107 Segment 3 and 4 015.jpg

AN107 Segment 3 and 4 016.jpg

Subdirectory Detector 3iDecember Breakdown\}

Section3B1HC1.JPG

Section3B1IIC2.JPG

Section3B3HC3.JPG

Section3B3HC4.JPG

Section3B3HC5.JPG

Section $3 \mathrm{C} 3 \mathrm{HCl}$.JPG

Section3C3HC2.JPG

Tree3-1.JPG

Tree3-2.JPG

Tree3-3.JPG

Tree3-4.JPG

Tree3-5.JPG

Tree3-6.JPG

Tree3-7.JPG

Tree3-8.JPG

Tree3-9.JPG

Subdirectory Detector 3'September Breakdown'

AN107 Segment 3 and 4 023.jpg

AN1 07 Segment 3 and 4 024.jpg

AN107 Segment 3 and 4 025.jpg

AN107 Segment 3 and 4 026.jpg

AN107 Scgment 3 and 4 027.jpg

AN107 Segment 3 and 4 028.jpg 
AN107 Segment 3 and 4 029.jpg AN107 Segment 3 and 4 030.jpg AN107 Segment 3 and 4 031.jpg AN107 Segment 3 and 4 032.jpg AN107 Segment 3 and 4 033.jpg AN 107 Segment 3 and 4 034.jpg AN107 Segment 3 and 4 035.jpg AN107 Segment 3 and 4 036.jpg AN107 Segment 3 and 4 037.jpg AN 107 Segment 3 and 4 038.jpg AN 107 Segment 3 and 4 039.jpg AN107 Segment 3 and 4 040.jpg ANI07 Segment 3 and 4 041.jpg AN 107 Segment 3 and 4 042.jpg AN 107 Segment 3 and 4 043.jpg AN 107 Segment 3 and 4 044.jpg AN107 Segment 3 and 4 045.jpg AN107 Segment 3 and 4 046.jpg AN 107 Segment 3 and 4 047.jpg AN 107 Segment 3 and 4 048.jpg AN 107 Segment 3 and 4 049.jpg AN107 Segment 3 and 4 050.jpg AN107 Segment 3 and 4 051.jpg AN107 Segment 3 and 4 052.jpg AN107 Segment 3 and 4 053.jpg AN107 Segment 3 and 4 054.jpg AN107 Segment 3 and 4 055.jpg AN107 Segment 3 and 4 056.jpg AN107 Segment 3 and 4 057.jpg AN107 Segment 3 and 4 058.jpg AN107 Segment 3 and 4 059.jpg AN107 Segment 3 and 4 060.jpg AN107 Segment 3 and 4 061.jpg AN107 Scgment 3 and 4 062.jpg

\author{
Subdirectory Detector 4\December Breakdown \\ Section4B1HC1.JPG \\ Section4B IHC2.JPG \\ Section4B3HC1.JPG \\ Section4B3HC2.JPG \\ Section4B3HC3.JPG \\ Section4C1HC2.JPG \\ Section4CIHC3.JPG \\ Section4C1HC4.JPG \\ Section4C1HC5.JPG \\ Section4C1HC6.JPG
}


Section4C1HC7.JPG

Section4C3HC1.JPG

Section4C3HC2.JPG

Section4C3HC3.JPG

Section4C.3HC4.JPG

Tree4-1.JPG

Tree4-2.JPG

Tree4-3.JPG

Tree4-4.JPG

Tree4-5.JPG

Tree4-6.JPG

Subdirectory Detector 4|September Breakdown

AN107 Segment 3 and 4 001.jpg

AN107 Segment 3 and 4 002.jpg

AN107 Segment 3 and 4 003.jpg

AN107 Segment 3 and 4 D04.jpg

AN107 Segment 3 and $4005 . j p g$

AN107 Segment 3 and 4 063.jpg

AN107 Segment 3 and 4 064.jpg

AN107 Segment 3 and 4 065.jpg

AN107 Segment 3 and 4 066.jpg

AN107 Segment 3 and 4 067.jpg

AN107 Segment 3 and 4 068.jpg

AN107 Segment 3 and 4 069.jpg

AN107 Segment 3 and 4 070.jpg

AN107 Segment 3 and 4 071.jpg

AN107 Segment 3 and 4 072.jpg

AN107 Segment 3 and 4 073.jpg

AN107 Segment 3 and 4 074.jpg

AN107 Segment 3 and 4 075.jpg

AN107 Segment 3 and 4 076.jpg

AN107 Segment 3 and 4 077.jpg

AN1 07 Segment 3 and 4 078.jpg

AN107 Segment 3 and 4 079.jpg

Chardocs: \AN107 Corrosion ProbelFinal Report \

Sample Analysis〈Macrophotography\

Subdirectory S06R001027A

AN107 Segment 1002

AN107 Segment 1003

AN107 Segment 1005

AN107 Segment 1006

AN107 Segment 1007

AN107 Segment 1008 
Subdirectory S06R001027B

AN107 Segment 1 base.jpg

AN107 Segment 1 C-Ring side A.jpg

AN107 Segment 1 C-Ring side B.jpg

AN107 Segment 1 top of C-ring crack.jpg

Subdirectory S06R001033

AN107 Segment 2 Stressed C-Ring notch.jpg AN107 Segment 2 Stressed C-Ring notch 2.jpg AN107 Segment 2 Stresscd C-Ring side A.jpg AN 107 Scgment 2 Stressed C-Ring bottom.jpd AN1 07 Scgment 2 Stressed C-Ring side B.jpg

Subdirectory S06R001036

AN107 Scgment 3 Bullet bottom.jpg AN107 Segment 3 Bullet.jpg

\section{Subdirectory S06R001038}

AN1 07 Segment 3 Unstressed C-Ring side A.jpg ANI 07 Segment 3 Unstressed C-Ring side B.jpg AN1 07 Segment 3 Unstressed C-Ring notch.jpg AN1 07 Segment 3 Unstressed C-Ring bottom.jpd

\section{Subdirectory S06R001039}

AN107 Segment 3 Stressed C-Ring bottom.jpg AN107 Segment 3 Stressed C-Ring side A1.jpg AN107 Segment 3 Stressed C-Ring side A.jpg AN107 Segment 3 Stressed C-Ring side B.jpg AN107 Segment 3 Stressed C-Ring notch 1.jpg AN107 Scgment 3 Stressed C-Ring notch 2.jpg AN107 Segment 3 Stressed C-Ring notch 3.jpg

\section{Subdirectory S06R001042}

AN107 Segment 4 Bullet bottoom 1.jpg AN107 Segment 4 Bullet bottoom2.jpg AN107 Segment 4 Bullet.jpg

\section{Subdirectory S06R001045}

AN107 Segment 4 Stressed C.Ring bottom.jpg AN107 Segment 4 Stressed C-Ring side A.jpg AN107 Segment 4 Stressed C-Ring side B.jpg AN107 Segment 4 Stressed C-Ring notch.jpg 


\title{
Chardocs: $\backslash A N 107$ Corrosion ProbelFinal Report \ Sample Analysis\Microphotography'
}

\author{
BlankClean-1a.jpg \\ BlankClean-1b.jpg \\ BlankClean-2a.jpg \\ BlankClean-2b.jpg \\ BlankClean-3a.jpg \\ BlankClcan-3b.jpg \\ BlankClcan-4a.jpg \\ BlankClcan-4b.jpg \\ BlankClcan-Sa.jpg \\ BlankClcan-5b.jpg \\ BlankClcan-6a.jpg \\ BlankClean-6b.jpg
}

Subdirectory BlanklCleanedtSEM

\section{Subdirectory BlankłUncleanedlOptical}

AN107Blank-10X1.JPG

AN107Blank-10X2.JPG

AN107Blank-20X1.JPG

AN107Blank-20X2.JPG

AN107Blank-40X1.JPG

\section{Subdirectory Blank $\backslash$ Uncleaned $\backslash S E M$}

AN107Blank-01a.JPG

AN107Blank-01b.JPG

AN107Blank-02a.JPG

AN 107Blank-02b.JPG

AN 107Blank-03a.JPG

AN107Blank-03b.JPG

AN107Blank-04a.JPG

AN107Blank-04b.JPG

AN107Blank-05.JPG

\author{
Subdirectory S06R001024\PItting\Optical \\ S06R001024Bullet-30Xa.JPG \\ S06R001024Bullet-63Xa.JPG \\ S06R001024Bullet-63Xb.JPG \\ S06R001 024Bullet-63Xc.JPG \\ S06R001024Bullet-63Xd.JPG \\ S06R001024Bullet-63Xe.JPG \\ S06R001024Bullet-63Xf.JPG \\ S06R001024Bullet-63Xg.JPG \\ S06R001024Bullet-63Xh.JPG \\ SO6R001024Bullet-63Xi.JPG
}


RPP-RPT-32425, Rev. 0

\begin{abstract}
S06R001024Bullet-63Xj.JPG
S06R001024Bullet-63Xk.JPG

S06R001024Bullet-63X1.JPG

S06R001024Bullet-63Xm.JPG

S06R001024Bullet-63Xn.JPG

S06R001 024Bullet-63Xo.JPG

S06R001024Bullet-63Xp.JPG
\end{abstract}

Subdirectory S06R001024|Pitting\SEM

S06R001024Bullet-1.jpg

S06R001024Bullet-10.jpg

S06R001024Bullet-10a.jpg

S06R001024Bullet-11.jpg

S06R001024Bullet-11a.jpg

S06R001024Bullet-12.jpg

S06R001024Bullet-12a.jpg

S06R001024Bullet-13.jpg

S06R001024Bullet-13a.jpg

S06R001024Bullet-14.jpg

S06R001024Bullet-14a.jpg

S06R001024Bullet-15.jpg

S06R001024Bullet-1 5a.jpg

S06R001024Bullet-1a.jpg

S06R001024Bullet-2.jpg

S06R001024Bullet-3.jpg

S06R001024Bullet-3a.jpg

S06R001024Bullet-4.jpg

S06R001024Bullet-4a.jpg

S06R001024Bullet-5.jpg

S06R001024Bullet-5a.jpg

S06R001024Bullet-6.jpg

S06R001024Bullet-6a.jpg

S06R001024Bullet-7.jpg

S06R001024Bullet-7a.jpg

S06R001024Bullet-8.jpg

S06R001024Bullet-8a.jpg

S06R001024Bullet-9.jpg

S06R001024Bullet-9a.jpg

Subdirectory S06R001024\Uncleaned $\backslash$ Optical

S06R001024-10X1.JPG

S06R001024-10X2.JPG

S06R001024-10X3.JPG

S06R001024-10X4.JPG

S06R001024-10X5.JPG

S06R001024-10X6.JPG 
RPP-RP'T-32425, Rev. 0

S06R001024-20X1.JPG

S06R001024-20X2.JPG

S06R001024-20X3.JPG

S06R001024-20X4.JPG

\author{
Subdirectory S06R001024\UncleanedWSEM \\ S06R001024-La.JPG \\ S06R001024-1b.JPG \\ SD6R001024-lc.JPG \\ SO6R001024-2a.JPG \\ SO6R001024-2b.JPG \\ S06R001024-2c.JPG
}

\author{
Subdirectory: S06R001027\Cleaned $\backslash$ Optical \\ S06R001027c-10X1.JPG \\ S06R001027c-10X2.JPG \\ S06R001027c-20X1.JPG \\ S06R001027c-20X2.JPG \\ S06R001027c-20X3.JPG \\ S06R001027e-20X4.JPG \\ S06R001027c-20X5.JPG \\ S06R001027c-40X1.JPG \\ S06R001027c-40X2.JPG \\ S06R001027C-40X3.JPG \\ S06R001027C-63X1.JPG \\ S06R001027C-63X2.JPG \\ S06R001027C-63X3.JPG \\ S06R001027C-63X4.JPG \\ Subdirectory S06R001027 Cleaned ISEM \\ S06R001027c-101a.JPG \\ S06R001027c-10lb.JPG \\ S06R001027c-102a.JPG \\ S06R001027c-102b.JPG \\ SO6R001027c-102c.JPG \\ S06R001027c-103a.JPG \\ SO6R001027c-103b.JPG \\ S06R001027c-104a.JPG \\ S06R001027c-104b.JPG \\ S06R001027c-105a.JPG \\ S06R001027c-105b.JPG \\ S06R001027c-106a.JPG \\ S06R001027c-106b.JPG \\ S06R001027c-107a.JPG \\ S06R001027c-107b.JPG \\ S06R001027c-108a.JPG
}


RPP-RPT-32425, Rev. 0

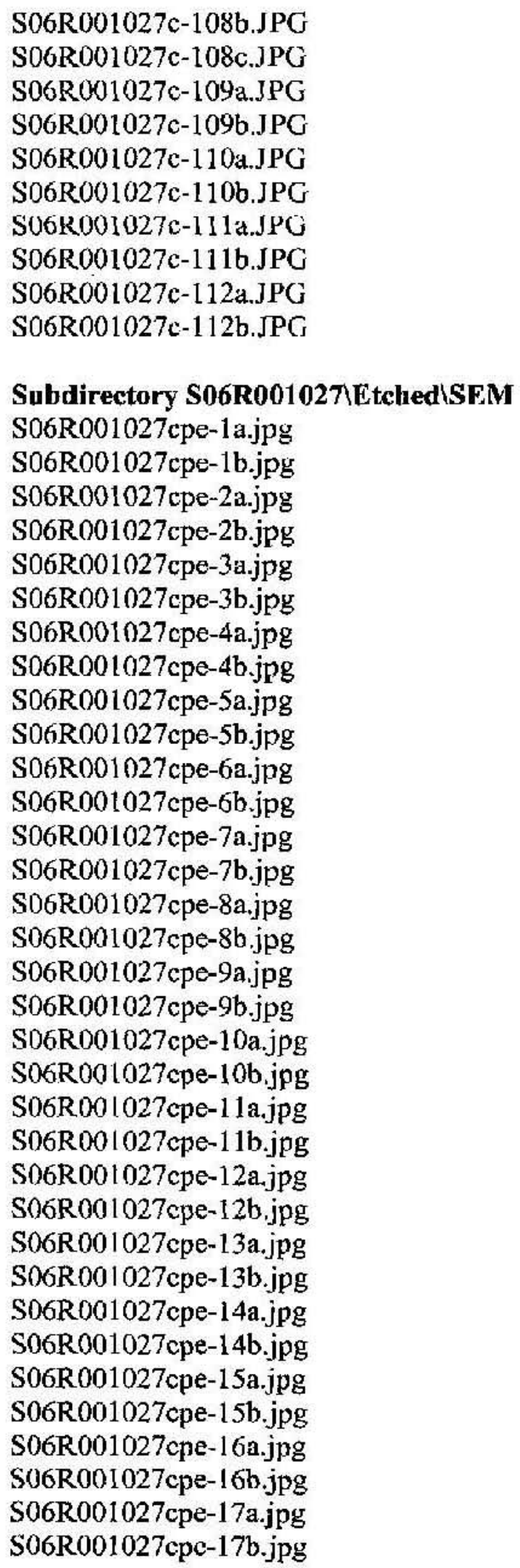


S06R001027cpe-18a.jpg S06R001027cpe-18b.jpg S06R001027cpe-19a.jpg S06R001027cpc-19b.jpg S06R001027cpc-20a.jpg S06R001027cpe-20b.jpg S06R001027cpc-21a.jpg S06R001027cpc-21b.jpg S06R001027cpe-22a.jpg S06R001027cpe-22b.jpg S06R001027cpc-23a.jpg S06R001027cpe-23b.jpg S06R001027cpe-24a.jpg S06R001027cpe-24b.jpg S06R001027cpe-25a.jpg S06R001027cpe-25b.jpg S06R001027cpe-26a.jpg S06R001027cpe-26b.jpg S06R001027cpe-27a.jpg S06R001027cpe-27b.jpg S06R001027cpe-28a.jpg S06R001027cpe-28b.jpg S06R001027cpe-29a.jpg S06R001027cpe-29b.jpg S06R001027cpe-30a.jpg S06R001027cpe-30b.jpg S06R001027cpe-31 a.jpg S06R001027cpe-31b.jpg S06R001027cpe-32a.jpg S06R001027cpe-32b.jpg

Subdirectory S06R001027Fractured 1 Optical S06R001027cca-15X1.JPG S06R001027cca-15X2.JPG S06R001027cca-40X1.JPG S06R001027ccb-15X1.JPG S06R001027ccb-15X2.JPG

Subdirectory S06R001027 7 ractured ISEM S06R001027cca-1a.JPG S06R001027cca-1b.JPG S06R001027cca-2a.JPG S06R001027cca-2b.JPG S06R001027cca-3a.JPG S06R001027cca-3b.JPG S06R001027cca-4a.JPG 
RPP-RPT-32425, Rev. 0

S06R001027cca-4b.JPG S06R001027cca-5a.JPG S06R001027cca-5b.JPG S06R001027cca-6a.JPG S06R001027cca-6b.JPG S06R001027cca-7a.JPG S06R001027cca-7b.JPG S06R001027cca-8a.JPG S06R001027 cca-8b.JPG S06R001027cca-9a.JPG S06R001027cca-9b.JPG S06R001027cca-10a.JPG S06R001027cca-10b.IPG S06R001027cca-11a.JPG S06R001027cca-11b.JPG SO6R001027cca-12a.JPG S06R001027cca-12b.JPG S06R001027cca-13a.JPG S06R001027cca-13b.JPG S06R001027cca-14a.JPG S06R001027ccb-1a.JPG S06R001027ccb-1b.JPG S06R001027ccb-2a.JPG S06R001027ccb-2b.JPG S06R001027ccb-3a.JPG S06R001027ccb-3b.JPG SO6R001027ccb-4a.JPG S06R001027ccb-4b.JPG S06R001027ccb-5a.JPG S06R001027ccb-Sb.JPG S06R001027ccb-6a.IPG S06R001027ccb-6b.JPG S06R001027ccb-7a.JPG S06R001027ccb-7b.JPG S06R001027ccb-8a.JPG S06R001027ccb-8b.JPG S06R001027ccb-9a.JPG S06R001027ccb-9b.JPG S06R001027ccb-10a.JPG S06R001027ccb-10b.JPG S06R001027ccb-11a.JPG S06R001027ccb-11b.JPG S06R001027ccb-12a.JPG S06R001027ccb-12b.JPG S06R001027ceb-13a.JPG S06R001027ccb-13b.JPG 
RPP-RP'T-32425, Rev. 0

S06R001027ccb-14a.JPG S06R001027ccb-14b.JPG S06R001027ccb-15a.JPG S06R001027ccb-15b.JPG S06R001027ccb-16a.JPG S06R001027ccb-16b.JPG S06R001027ccb-17a.JPG S06R001027ccb-17b.JPG S06R001027ccb-18a.JPG S06R001027ccb-18b.JPG

Subdirectory S06R001027 Pitting $\backslash$ Ptical S06R001027Base-10Xa.JPG S06R001027Base-30Xa.JPG S06R001027Base-63Xa.JPG S06R001027Base-63Xb.JPG S06R001027Base-63Xc.JPG S06R001027Crevice-63Xa.JPG S06R001027Crevice-63Xb.JPG

S06R001027Crevice-63Xc.JPG

\section{Subdireetory S06R001027)Pitting/SEM}

S06R001027Base-1.jpg S06R001027Base-la.jpg S06R001027Base-2.jpg S06R001027Base-2a.jpg S06R001027Base-3.jpg S06R001027Base-3a.jpg S06R001027Crevice-1.jpg S06R001027Crevice-1a.jpg S06R001027Crevice-2.jpg S06R001027Crevice-2a.jpg S06R001027Crevice-3.jpg S06R001027Crevice-3a.jpg

Subdirectory S06R001027Polished $\backslash$ Optical SO6R001027cp-20X1.JPG S06R001027cp-20X2.JPG S06R001027cp-20X3.JPG S06R001027cp-40XI.JPG

Subdirectory S06R00t027Polished ISFM S06R001027cp-1a.JPG S06R001027cp-1b.JPG S06R001027cp-2a.JPG S06R001027cp-2b.JPG 
S06R001027cp-3a.JPG S06R001027cp-3b.JPG S06R001027cp-4a.JPG S06R001027cp-4b.JPG S06R001027cp-5a.JPG S06R001027cp-5b.JPG SU6R001027cp-6a.JPG S06R001027cp-6b.JPG S06R001027cp-7a.JPG S06R001027cp-7b.JPG S06R001027cp-8a.JPG S06R001027cp-8b.JPG S06R001027cp-9a.JPG S06R001027cp-9b.JPG S06R001027cp-10a.JPG S06R001027cp-10b.JPG S06R001027cp-11a.JPG S06R001027ep-11b.JPG S06R001027cp-12a.JPG S06R001027cp-12b.JPG S06R001027cp-13a.JPG S06R001027cp-13b.JPG S06R001027cp-14a.JPG S06R001027cp-14b.JPG S06R001027cp-15a.JPG S06R001027cp-15b.JPG S06R001027cp-16a.JPG S06R001027cp-16b.JPG S06R001027cp-17a.JPG S06R001027cp-17b.JPG S06R001027cp-18a.JPG S06R001027cp-18b.JPG S06R001027cp-19a.JPG S06R001027cp-19b.JPG S06R001027cp-20a.JPG S06R001027cp-20b.JPG S06R001027cp-21a.JPG S06R001027cp-21b.JPG

Subdirectory S06R001027Uncleaned $\backslash$ Optical Side A S06R001027-10X1.JPG S06R001027-10X2.JPG S06R001027-10X3.JPG S06R001027-20X1.JPG S06R001027-63X1.JPG S06R001027-63X2.JPG 
RPP-RPT-32425, Rev, 0

S06R001027-63X3.JPG

S06R001027-63X4.JPG

Subdirectory S06R001027 UncleanediOpticaliside B

S06R001027-10X6.JPG

S06R001027-10X7.JPG

S06R001027-20X2.JPG

S06R001027-20X3.JPG

S06R001027-63X5.JPG

S06R001027-63X6.JPG

Subdirectory S06R001027Uncleaned $\backslash$ Optical $\backslash$ Top

S06R001027-10X4.JPG

S06R001027-10X5.JPG

\section{Subdirectory S06R001027 UncleanedISEM}

S06R001027A-1 a.jpg

SO6R001027A-1b.jpg

S06R001027A-2a.jpg

S06R001027A-2b.jpg

SOGRD01027A-3a.jpg

SOGR001027A-3b.jpg

S06R001027A-4a.jpg

S06R001027A-4b.jpg

S06R001027A-5a.jpg

SO6R001027A-5b.jpg

S06R001027A-6a.jpg

S06R001027A-6b.jpg

S06R001027A-7a.jpg

S06R001027A-7b.jpg

S06R001027A-8a.jpg

S06R001027A-8b.jpg

S06R001027A-9a.jpg

S06R001027A-9b.jpg

S06R001027A-10a.jpg

S06R001027A-10b.jpg

S06R001027A-1 la.jpg

S06R001027A-11b.jpg

S06R001027A-12a.jpg

S06R001027A-12b.jpg

S06R001027A-13a.jpg

S06R001027A-13b.jpg

S06R001027A-14a.jpg

S06R001027A-14b.jpg

S06R001027A-15a.jpg

SO6R001027A-15b.jpg 
RPP-RPT-32425, Rev. 0

S06R001027A-16a.jpg S06R001027A-16b.jpg S06R001027A-17a.jpg S06R001027A-17b.jpg S06R001027A-18a.jpg S06R001027A-18b.jpg S06R001027A-19a.jpg S06R001027A-19b.jpg S06R01027A-20a.jpg S06R001027A-20b.jpg S06R001027A-2la.jpg S06R001027A-21b.jpg S06R001027A-22a.jpg S06R001027A-22b.jpg S06R001027A-23a.jpg S06R001027A-23b.jpg S06R001027A-24a.jpg S06R001027A-24b.jpg S06R001027A-25a.jpg S06R001027A-25b.jpg S06R001027A-26a.jpg S06R001027A-26b.jpg S06R001027A-26c.jpg S06R001027A-27a.jpg S06R001027A-27b.jpg 506R001027A-27c.jpg S06R001027A-28a.jpg S06R001027A-28b.jpg S06R001027A-28c.jpg S06R001027A-29a.jpg S06R001027A-29b.jpg S06R001027A-29c.jpg S06R001027A-30a.jpg S06R001027A-30b.jpg SU6R001027A-30c.jpg S06R001027A-31a.jpg S06R001027A-31b.jpg S06R001027A-31c.jpg S06R001027A-32a.jpg S06R001027A-32b.jpg S06R001027A-32c.jpg S06R001027B-40a.JPG S06R001027B-40b.JPG S06R001027B-40c.JPG S06R001027B-41a.JPG S06R001027B-41b.JPG 
RPP-RPT-32425, Rev. 0

S06R001027B-41c.JPG

S06R001027B-42a.JPG

S06R001027B-42b.JPG

Subdirectory S06R001028łcleanedISEM

S06R001028c-1a.JPG

S06R001028c-1b.JPG

S06R001028c-2a.JPG

S06R001028c-2b.JPG

Subdirectory S06R001028\pitting $\mid$ Optical

S06R001028Bolt-10X1.JPG

S06R001028Bolt-10X2.JPG

S06R001028Bolt-10X3.JPG

S06R001028Bolt-10X4.JPG

S06R001028Bolt-20X1.JPG

S06R001028Bolt-20X2.JPG

S06R001028Bolt-30X1.JPG

S06R001028Bolt-30X2.JPG

S06R001028Bolt-30X3.JPG

S06R001028Bolt-30X4.JPG

S06R001028Bolt-30X5.JPG

S06R001028Bolt-30X6.JPG

S06R001028Bolt-30X7.JPG

S06R001028Bolt-63X1.JPG

S06R001028Bolt-63X2.JPG

S06R001028Bolt-63X3.JPG

S06R001028Bolt-63X4.JPG

S06R001028Bolt-63X5.JPG

S06R001028Bolt-63X6.JPG

S06R001028Bolt-63X7.JPG

Subdirectory $\mathbf{S 0 6 R 0 0 1 0 2 8}$ \pittinglSEM

SO6RD01028Base-14.jpg

SO6R001028Base-14a.jpg

S06R001028Base-15.jpg

S06R001028Bolt-1.jpg

SO6RD01028Bolt-10.jpg

S06R001028Bolt-10aBSED.jpg

S06R001028Bolt-10b.jpg

S06R001028Bolt-11.jpg

S06R001028Bolt-1a.jpg

S06R001028Bolt-2.jpg

S06R001028Bolt-2a.jpg

S06R001028Bolt-3.jpg

S06R001028Bolt-3a.jpg 
S06R001028Bolt-3b.jpg S06R001028Bolt-4.jpg S06R001028Bolt-4a.jpg S06R001028Bolt-4b.jpg S06R001028Bolt-5.jpg S06R001028Bolt-5a.jpg S06R001028Bolt-5b.jpg S06R001028Bolt-6.jpg S06R001028Bolt-6a.jpg S06R001028Bolt-7.jpg S06R001028Bolt-7a.jpg S06R001028Bolt-8.jpg S06R001028Bolt-8a.jpg S06R001028Bolt-9.jpg S06R001028Rim-12.jpg S06R001028Rim-12a.jpg S06R001028Rim-13.jpg S06R001028Rim-13a.jpg

\footnotetext{
Subdirectory S06R001033 ifractured ISEM

S06R001033cca-la.jpg S06R001033cca-1b.jpg S06R001033cca-2a.jpg SO6R001033cca-2b.jpg S06R001033cca-3a.jpg S06R001033cca-3b.jpg S06R001033cca-4a.jpg S06R001033cca-4b.jpg S06R001033cca-4c.jpg S06R001033cca-5a.jpg S06R001033cca-5b.jpg S06R001033cca-5c.jpg S06R001033cca-6a.jpg S06R001033cca-6b.jpg S06R001033cca-6c.jpg S06R001033cca-7a.jpg S06R001033cca-7b.jpg S06R001033ccu-8a.jpg S06R001033cca-8b.jpg S06R001033cca-9a.jpg S06R001033cca-9b.jpg S06R001033cca-10a.jpg S06R001033cca-10h.jpg SO6R001033cca-11a.jpg SO6R001033cca-11b.jpg S06R001033cca-12a.jpg
} 
RPP-RPT-32425, Rev. 0

RPP-RPT-32425, Rev. 0

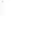

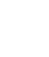


RPP-RPT-32425, Rev. 0

\author{
Subdirectory S06R001042\Pitting|Optical \\ S06R001042Base-20Xa.JPG \\ S06R001042Base-20Xb.JPG \\ S06R001042Base-20Xc.JPG \\ S06R001042Base-30Xa.JPG \\ S06R001042Base-63Xa.JPG \\ S06R001042Base-63Xb.JPG \\ S06R001042Base-63Xe.JPG \\ S06R001042Base-63Xd.JPG \\ S06R001042Base-63Xe.JPG \\ S06R001042Rim-63Xa.JPG \\ S06R001042Rim-63Xb.JPG \\ S06R001042Rim-63Xc.JPG \\ Subdirectory S06R001042\PittingISFM \\ S06R001042base-1.jpg \\ S06R001042base-1a.jpg \\ S06R001042base-2.jpg \\ S06R001042base-2a.jpg \\ S06R001042base-3.jpg \\ S06R001042base-3a.jpg \\ S06R001042base-4.jpg \\ S06R001042base-4a.jpg \\ S06R001042base-5.jpg \\ S06R001042base-5a.jpg \\ S06R001042base-6.jpg \\ S06R001042base-6a.jpg \\ S06R001042base-7.jpg \\ S06R001042base-7a.jpg \\ S06R001042base-8.jpg \\ S06R001042base-8a.jpg \\ S06R001042rim-1.jpg \\ S06R001042rim-1a.jpg \\ S06R001042rim-2.jpg \\ S06R001042rim-2a.jpg \\ SO6R001042rim-3.jpg \\ S06R001042rim-3a.jpg \\ Subdirectory S06R001042 UncleanedISEM \\ S06R001042-1a.jpg \\ S06R001042-1b.jpg \\ S06R001042-2a.jpg \\ S06R001042-2b.jpg \\ S06R001042-3a.jpg \\ S06R001042-3b.jpg \\ S06R001042-4a.jpg
}


RPP-RPT-32425, Rev. 0

\begin{abstract}
S06R001042-4b.jpg
S06R001042-5a.jpg

S06R001042-5b.jpg

S06R001042-6a.jpg

S06R001042-6b.jpg

S06R001042-6c.jpg

S06R001042-7a.jpg

S06R001042-7b.jpg

S06R001042-7c.jpg

S06R001042-8a.jpg

S06R001042-8b.jpg

S06R001042-8c.jpg
\end{abstract}

\title{
Subdirectory S06R001045tEtchediOptical \\ S06R001045cpe-30X1.JPG \\ S06R001045cpe-63X1.JPG \\ S06R001045cpe-63X2.JPG
}

\author{
Subdirectory S06R001045ไEtchedISEM \\ S06R001045cpe-1a.jpg \\ S06R001045cpe-1b.jpg \\ S06R001045cpe-2a.jpg \\ SOGR001045cpe-2b.jpg \\ S06R001045cpe-3a.jpg \\ S06R001045cpe-3b.jpg \\ SO6R001045cpe-4a.jpg \\ SO6R001045cpe-4h.jpg \\ S06R001045cpe-5a.jpg \\ S06R001045cpe-5b.jpg \\ S06R001045cpe-6a.jpg \\ S06R001045cpe-6b.jpg \\ S06R001045cpe-7a.jpg \\ SO6RD01045cpe-7b.jpg \\ S06R001045cpe-8a.jpg \\ S06R001045cpe-8b.jpg \\ S06R001045cpe-9a.jpg \\ SO6R001045cpe-9b.jpg \\ S06R001045cpe-10a.jpg \\ S06R001045cpe-10b.jpg \\ S06R001045cpe-I1a.jpg \\ S06R001045cpe-11b.jpg \\ S06R001045cpe-12a.jpg \\ S06R001045cpe-12b.jpg \\ S06R001045cpe-13a.jpg \\ S06R001045cpe-13b.jpg \\ S06R001045cpc-14a.jpg
}




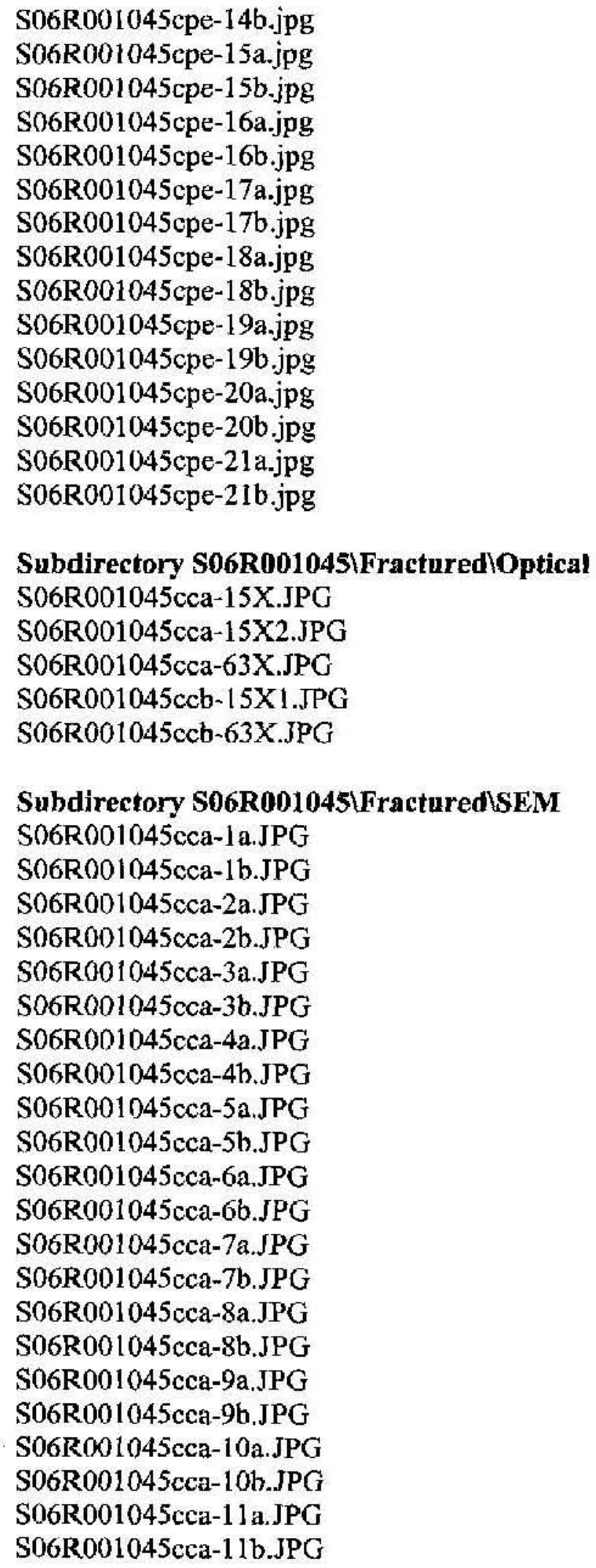


S06R001045cca-12a.JPG S06R001045cca-12b.JPG S06R001045cca-13a.JPG S06R001045cca-13b.JPG S06R001045cca-14a.JPG S06R001045cca-14b.JPG S06R001045cca-15a.JPG S06R001045cca-15b.JPG S06R001045cca-16a.JPG S06R001045cca-16b.JPG S06R001045cca-17a,JPG S06R001045cca-17b.JPG S06R001045cca-18a.JPG S06R001045cca-18b.JPG S06R001045cca-19a.JPG S06R001045ccb-10a.JPG S06R001045ccb-10b.JPG S06R001045ccb-11a.JPG S06R001045ccb-1 lb.JPG S06R001045ceb-12a.JPG S06R001045ccb-12b.JPG S06R001045ccb-13a.JPG S06R001045ccb-13b.JPG S06R001045ccb-14a.JPG S06R001045ccb-14b.JPG S06R001045ccb-15a.JPG S06R001045ccb-15b.JPG S06R001045ccb- I6a.JPG S06R001045ccb-16b.JPG S06R001045ccb-17a.JPG S06R001045ccb-17b.JPG S06R001045ccb-1a.JPG S06R001045cch-1b.JPG S06R001045ccb-2a.JPG S06R001045ccb-2b.JPG S06R001045ccb-3a.JPG S06R001045ccb-3b.JPG S06R001045ccb-4a.JPG S06R001045cch-4b.JPG S06R001045ccb-5a.JPG S06R001045ccb-5b.JPG S06R001045ccb-6u.JPG S06R001045ccb-6b.JPG S06R001045ccb-7a.JPG S06R001045ccb-7b.JPG S06R001045ccb-8a.JPG 
RPP-RPT-32425, Rev, 0

S06R001045ccb-8b.JPG

S06R001045ccb-9a.JPG

S06R001045ccb-9b.JPG

Subdirectory S06R001045 Pitting $\backslash$ Optical

S06R001045Base-63Xa.JPG

S06R001045Base-63Xb.JPG

S06R001045Crevice-20Xa.JPG

S06R001045Crevice-20Xb.JPG

Subdirectory S06R001045 \Pitting ISEM

S06R001045Base-1.JPG

S06R001045Base-1a.JPG

S06R001045Base-2.JPG

S06R001045Base-2a.JPG

S06R001045Base-3.JPG

S06R001045Base-3a.JPG

S06R001045Crevice-1.JPG

S06R001045Crevice-1a.JPG

S06R001045Crevice-2.JPG

S06R001045Crevice-2a.JPG

S06R001045Crevice-3.JPG

S06R001045Crevice-3a.JPG

S06R001045Crevice-4.JPG

S06R001045Crevice-4a.JPG

S06R001045Crevice-5.JPG

S06R001045Crevice-5a.JPG

S06R001045Rim-1.JPG

S06R001045Rim-1a.JPG

S06R001045Rim-2.JPG

S06R001045Rim-2a.JPG

S06R001045Rim-3.JPG

S06R001045Rim-3a.JPG

Subdirectory S06R001045\PolishediSEM

S06R001045cp-la.jpg

S06R001045cp-lb.jpg

S06R001045cp-2a.jpg

S06R001045cp-2b.jpg

S06R001045cp-3a.jpg

S06R001045cp-3b.jpg

S06R001045cp-4a.jpg

S06R001045cp-4b.jpg

S06R001045cp-5a.jpg

S06R001045cp-5b.jpg

S06R001045cp-6a.jpg 


\begin{abstract}
SO6R001045cp-6b.jpg SO6R001045cp-7a.jpg S06R001045cp-7b.jpg S06R001045cp-8a.jpg S06R001045cp-8b.jpg S06R001045cp-9a.jpg S06R001045cp-9b.jpg SO6R001045cp-10a.jpg S06R001045cp-10b.jpg S06R001045cp-11a.jpg SO6R001045cp-11b.jpg S06R001045cp-12a.jpg SO6R001045cp-12b.jpg SO6R001045cp-12c.jpg SD6R001045cp-13a.jpg SO6R001045cp-13b.jpg
\end{abstract}

Subdirectory S06R001045/UncleanediOptical S06R001045-20X1.JPG SO6R001045-20X2.JPG SO6R001045-20X3.JPG S06R001045-20X4.JPG S06R001045-20X5.JPG S06R001045-20X5Flip.JPG

S06R001045-20X6.JPG S06R001045-20X7.JPG S06R001045-20X8,JPG S06R001045-20X9.JPG

Subdirectory SD6R001045IUncleaned ISEM S06R001045-1a.jpg S06R001045-1b.jpg S06R001045-2a.jpg S06R001045-2b.jpg S06R001045-3a.jpg S06R001045-3b.jpg S06R001045-4a.jpg S06R001045-4b.jpg S06R001045-5a.jpg S06R001045-5b.jpg S06R001045-6a.jpg S06R001045-6b.jpg S06R001045-7a.jpg S06R001045-7b.jpg S06R001045-8a.jpg S06R001045-8b.jpg 


\author{
S06R001045-9a.jpg \\ S06R001045-9b.jpg \\ S06R001045-10a.jpg \\ S06R001045-10b.jpg \\ S06R001045-11a.jpg \\ S06R001045-11b.jng \\ S06R001045-11c.jpg \\ S06R001045-12a.jpg \\ S06R001045-12b.jpg \\ S06R001045-13a.jpg \\ S06R001045-13b.jpg \\ SO6R001045-13c.jpg \\ S06R001045-14a.jpg \\ S06R001045-14b.jpg \\ S06R001045-14c.jpg \\ S06R001045-15a.jpg \\ S06R001045-15b.jpg \\ S06R001045-15c.jpg \\ S06R001045-16a.jpg \\ S06R001045-16b.jpg \\ S06R001045-16c.jpg \\ S06R001045-17a.jpg \\ S06R001045-17b.jpg \\ S06R001045-17c.jpg \\ S06R001045-18a.jpg \\ S06R001045-18b.jpg \\ S06R001045-18c.jpg \\ S06R001045-19a.jpg \\ S06R001045-19b.jpg \\ S06R001045-19c.jpg \\ SO6R001045-20a.jpg \\ S06R001045-20b.jpg \\ S06R001045-21a.jpg \\ S06R001045-21b.jpg \\ S06R001045-2lc.jpg

\section{Standards} \\ mesh40um-63Xa.JPG \\ Ruler 1mm-10Xa.JPG \\ Ruier1mm-20Xa.JPG \\ RulerImm-30Xa.JPG \\ Ruler1mm-40Xa.JPG \\ Ruler1mm-63Xa.JPG \\ Size40um-1.jpg \\ Size40um-10.jpg \\ Size40um-11.jpg
}


RPP-RPT-32425, Rev. 0

Size40um-12.jpg

Size40um-13.jpg

Size40um-14.jpg

Size40um-15.jpg

Size40um-16.jpg

Size40um-2.jpg

Size 40um-3.jpg

Size40um-4.jpg

Size40um-5.jpg

Size40um-6.jpg

Size40um-7.jpg

Sizc40um-8.jpg

Sizc40um-9.jpg 


\section{DISTRIBUTION SHEET}

To

Distribution

Project Title/Work Order

Finai Analytical Rcsults from the Examination of Corrosion on Scctions of Corrosion Probe kemoved from Jank 241-AN-107....

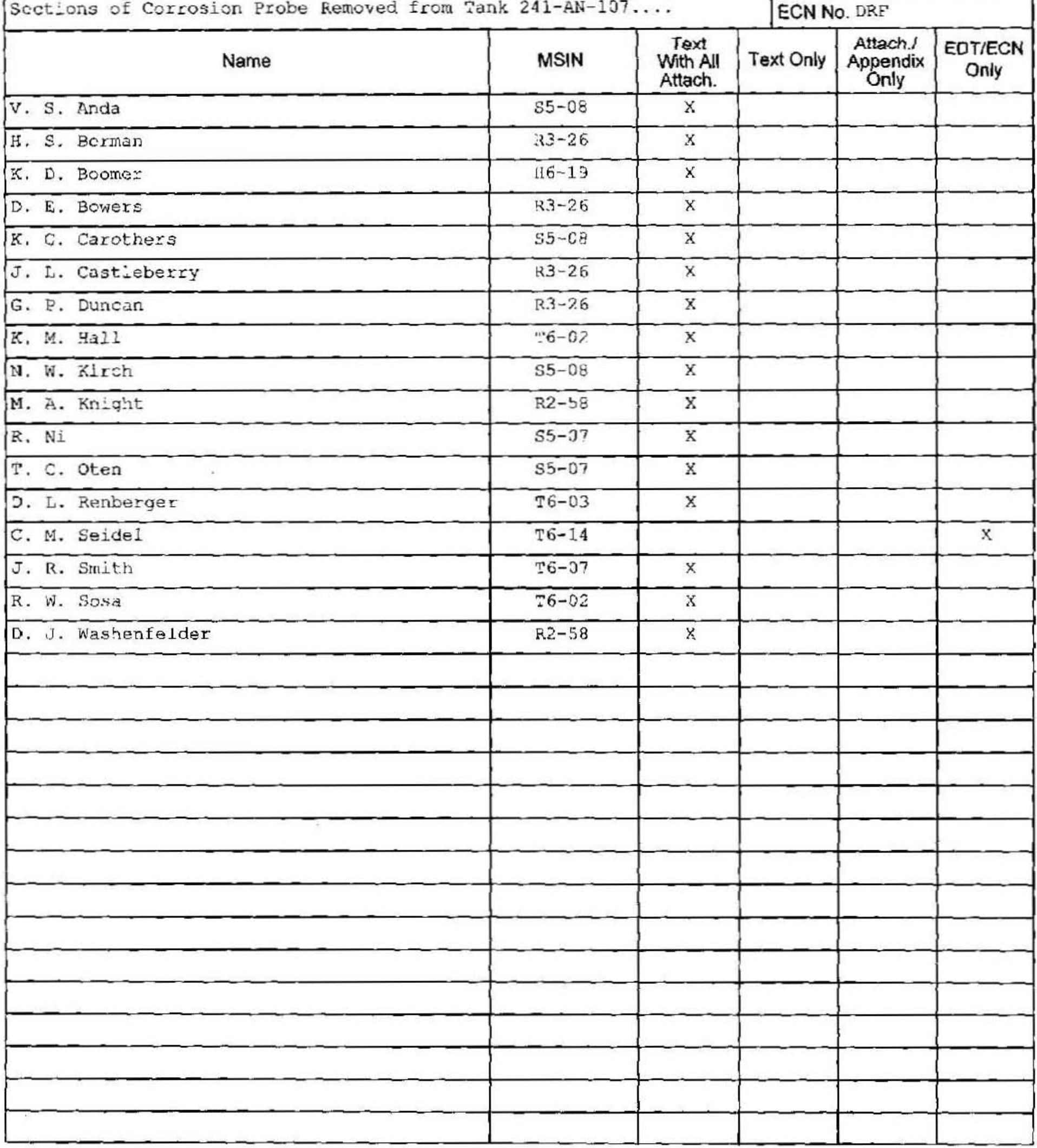

From

G. A. Cooke and J. B. Duncan

Page 1 of 1

Date Narch 2007

EDT NO. DRF

NORE

ECN No. DRF

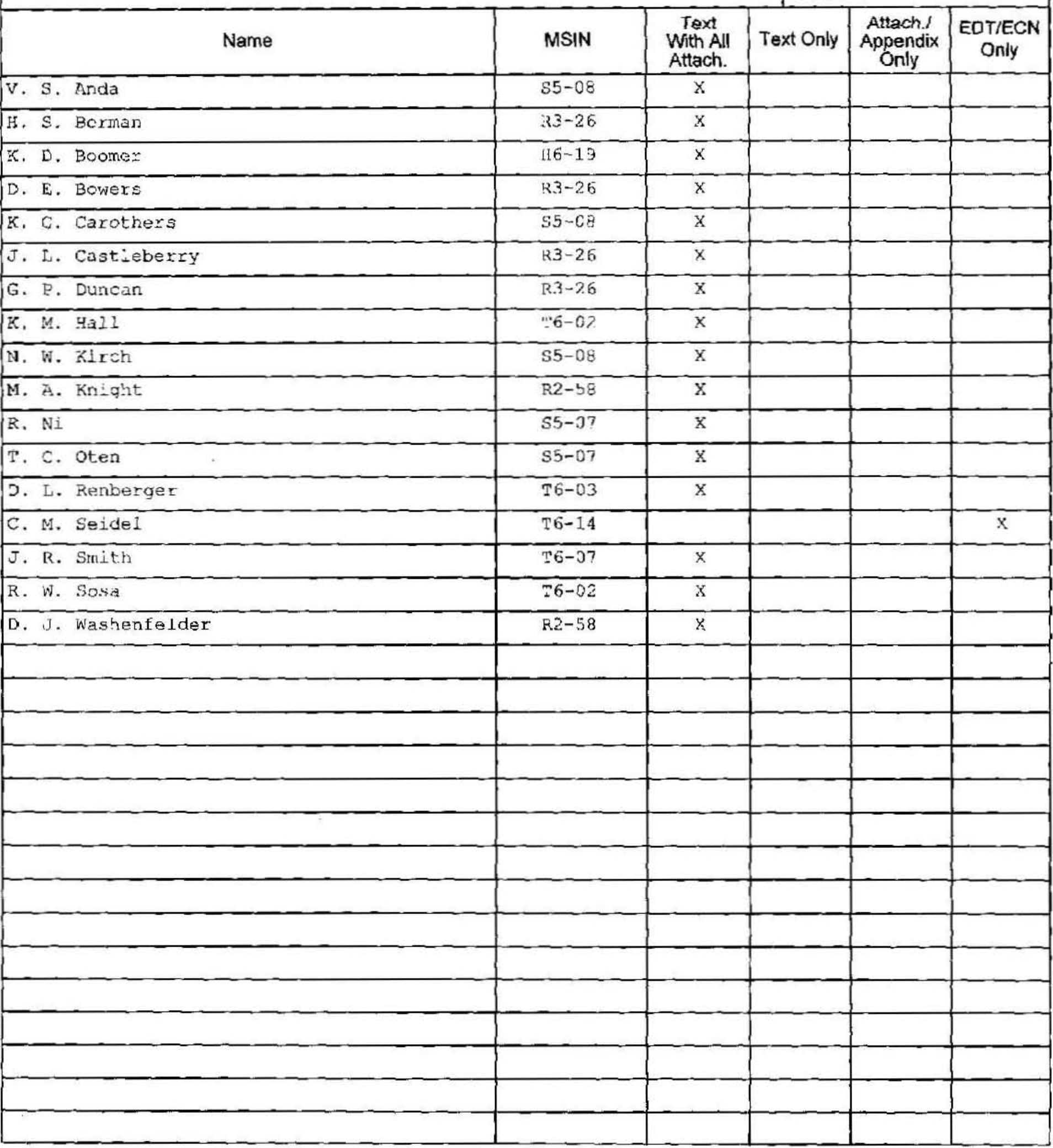

